\title{
A NEW APPROACH FOR THE PREDICTION OF BRITTLENESS INDEX BASED ON CHEMICAL PROPERTIES OF BASALTIC ROCKS
}

\author{
Candan BILEN ${ }^{1)}$ *, Selman ER ${ }^{2)}$, Atiye TUĞRUL ${ }^{3)}$ and Murat YILMAZ ${ }^{3)}$ \\ 1) Department of Geological Engineering, Zonguldak Bulent Ecevit University, Zonguldak, Turkey \\ 2) Department of Geography, Inonu University, Malatya, Turkey \\ 3) Department of Geological Engineering, Istanbul University-Cerrahpasa, Istanbul, Turkey
}

*Corresponding author's e-mail: candanalptekin@gmail.com

\begin{tabular}{|c|c|}
\hline ARTICLE INFO & ABSTRACT \\
\hline Article history: & \multirow{9}{*}{$\begin{array}{l}\text { Rock brittleness is one of the most important issues in rock mechanics. There is not yet an available } \\
\text { method for defining or measuring directly the rock brittleness. The aim of this study is to suggest } \\
\text { a new chemical index parameter for the prediction of basaltic rocks' brittleness. In the order of } \\
\text { that abovementioned purpose, a total of } 23 \text { basaltic rock samples were collected from different } \\
\text { region of Turkey. Samples were initially tested to determine their chemical properties. Then, } \\
\text { mechanical tests were carried out to define the brittleness indices (B1, B2, and B3) for each } \\
\text { corresponding sample. Finally, relations between parameters obtained from test results and } \\
\text { brittleness indices were examined with regression analysis. According to the results obtained, } \\
\text { a new chemical parameter (CP) was proposed for predicting brittleness via major oxide element } \\
\text { components of basaltic rocks. It was found out that, B1 and B2 are not reliable parameters for } \\
\text { predicting the different properties, however; B3 and CP can be employed as good criteria } \\
\text { for predicting the different properties of basaltic rocks (especially in terms of chemical and } \\
\text { mechanical properties). }\end{array}$} \\
\hline Received 18 January 2021 & \\
\hline Accepted 30 April 2021 & \\
\hline Available online 12 May 2021 & \\
\hline Keywords: & \\
\hline Basaltic rocks & \\
\hline Chemical composition & \\
\hline Brittleness & \\
\hline Mechanical properties & \\
\hline
\end{tabular}

\section{INTRODUCTION}

The brittleness is generally contemplated as one of the most important mechanical properties of rock. Rock brittleness is one of the most popular research areas in rock mechanics and this is because some rocks show brittle behavior when exposed to different type of loads. Brittleness characteristics as important geotechnical parameters which are used in rock engineering projects such as tunneling and underground openings, dam foundations, drilling, and slope stability analysis (Aligholi et al., 2017). The brittleness index (BI) is a term that is usually used to quantify the brittleness of rock mass. The uniaxial compressive strength (UCS) and Brazilian tensile strength (BTS) are two main geomechanical properties of rock that can be used for the brittleness index (BI) prediction (Zhang et al., 2016).

An excessive number of different measures of rock brittleness can be found in the literature. For example, Yagiz (2009) have tried to evaluate the rock brittleness with punch penetration test and he has tried to predict rock brittleness in terms of a function based on uniaxial compressive strength, Brazilian tensile strength and density of rock. Although Yagiz (2009) stated the fact that "there is no confident way to measure rock brittleness directly", same author has suggested the punch penetration test in the order of direct measurement of rock toughness and brittleness
(Yagiz, 2006). In this context, Yagiz and Karahan (2015) have employed the same data of rock brittleness derived from punch penetration test in order to understand TBM (tunnel boring machine) performance. Although many researchers have tried to define rock brittleness indirectly, there is no yet an agreement to describe or measure it (Yagiz, 2009). This abovementioned shortage of rock brittleness description is also explained by Kaunda and Asbury (2016). According to Kaunda and Asbury (2016), strength based methods to describe the rock brittleness under various load conditions and anisotropies are not sufficient and they seem to fall short in describing rock brittleness. In their study of Kaunda and Asbury (2016) have tried to predict rock brittleness by employing the data based on P- and S-wave velocities, elastic properties and rock type. Some other researchers like Guo et al. (2012) however tried to describe rock brittleness based on mineralogy and porosity in shale (non-strength based rock physics). In addititon to these abovementioned attempts to describe rock brittleness, Yagiz and Gokceoglu (2010) have employed nonlinear regression models and fuzzy logic to predict rock brittleness. In this context, a summary of literature descriptions of rock brittleness is provided in Table 1 (adapted from the study of Zhang et al. (2021)). 
Table 1 Summary of literature description of rock brittleness (Adapted from the study of Zhang et al., 2021).

\begin{tabular}{|c|c|c|c|}
\hline Equation No & Suggested method for the description of rock brittleness & Note* & Reference \\
\hline [1] & $\mathrm{b}_{1}=\sigma_{\mathrm{c}} / \sigma_{\mathrm{t}}$ & $*_{\mathrm{i}}$ & Hucka and Das (1974) \\
\hline [2] & $\mathrm{b}_{2}=\sigma_{\mathrm{c}} \sigma_{\mathrm{t}} / 2$ & $*_{\mathrm{i}}$ & Altindag (2003) \\
\hline [3] & $\mathrm{b}_{3}=\left(\sigma_{\mathrm{c}} \sigma_{\mathrm{t}} / 2\right)^{0.5}$ & $*_{\mathrm{i}}$ & Altindag (2003) \\
\hline [4] & $\mathrm{b}_{4}=\left(\sigma_{\mathrm{c}}-\sigma_{\mathrm{t}}\right) /\left(\sigma_{\mathrm{c}}+\sigma_{\mathrm{t}}\right)$ & $*_{\mathrm{i}}$ & Hucka and Das (1974) \\
\hline [5] & $\mathrm{b}_{5}=\sigma_{\mathrm{c}} / \sigma_{\mathrm{t}}=8 \sigma_{\mathrm{c}} / \sigma_{\mathrm{ci}}=8 / \mathrm{K}$ & $*_{\mathrm{i}}$ & Wang et al. (2014) \\
\hline [6] & $\mathrm{b}_{6}=\left(\sigma_{\mathrm{c}}-\sigma_{\mathrm{t}}\right) /\left(\sigma_{\mathrm{c}}+\sigma_{\mathrm{t}}\right)=(8-\mathrm{K}) /(8+\mathrm{K})$ & $*_{\mathrm{i}}$ & Wang et al. (2014) \\
\hline [7] & $\mathrm{b}_{7}=\left(\tau_{\mathrm{p}}-\tau_{\mathrm{r}}\right) / \tau_{\mathrm{p}}$ & $*_{\mathrm{i}}$ & Bishop (1967) \\
\hline [8] & $\mathrm{b}_{8}=\varepsilon_{\mathrm{r}} / \varepsilon_{\mathrm{t}}$ & $*_{\mathrm{ii}}$ & Hucka and Das (1974) \\
\hline [9] & $\mathrm{b}_{9}=\varepsilon_{\text {ini }} / \varepsilon_{\mathrm{t}}$ & $*_{\mathrm{ii}}$ & \\
\hline [10] & $\mathrm{b}_{10}=\left(\varepsilon_{\mathrm{p}}-\varepsilon_{\mathrm{r}}\right) / \varepsilon_{\mathrm{p}}$ & $*_{\mathrm{ii}}$ & Bishop (1967) \\
\hline [11] & 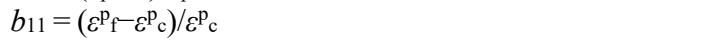 & $*_{\mathrm{ii}}$ & Hajiabdolmajid and Kaiser (2003) \\
\hline [12] & $\mathrm{b}_{12}=\varepsilon_{11} \times 100 \%$ & $*_{\mathrm{ii}}$ & Andreev (1995) \\
\hline [13] & $\mathrm{b}_{13}=\left(\tau_{\mathrm{p}}-\tau_{\mathrm{r}}\right) / \tau_{\mathrm{p}} \cdot \lg \mid \mathrm{k}_{\mathrm{ac}} / 10$ & $*_{\mathrm{iii}}$ & Meng et al. (2015) \\
\hline [14] & $b_{14}=\left(\sigma_{p}-\sigma_{r}\right) /\left(\varepsilon_{r}-\varepsilon_{p}\right)+\left[\left(\sigma_{p}-\sigma_{r}\right)\left(\varepsilon_{r}-\varepsilon_{p}\right)\right] /\left(\sigma_{p} \varepsilon_{p}\right)$ & *iii & Xia et al. (2016) \\
\hline$[15]$ & $\mathrm{b}_{15}=\left[\left(\sigma_{\mathrm{p}}-\sigma_{\mathrm{i}}\right) / \sigma_{\mathrm{p}}\right] /\left(\varepsilon_{\mathrm{p}}-\varepsilon_{\mathrm{i}}\right)+\left[\left(\sigma_{\mathrm{p}}-\sigma_{\mathrm{r}}\right) / \sigma_{\mathrm{p}}\right] /\left(\varepsilon_{\mathrm{r}}-\varepsilon_{\mathrm{p}}\right)$ & *iii & Chen et al. (2019a) \\
\hline$[16]$ & $\mathrm{b}_{16}=\mathrm{W}_{\mathrm{r}} / \mathrm{W}_{\mathrm{t}}$ & $*_{\text {iv }}$ & Hucka and Das (1974) \\
\hline [17] & $\mathrm{b}_{17}=\mathrm{dW}_{\mathrm{r}} / \mathrm{dW}_{\mathrm{e}}$ & $*_{\text {iv }}$ & Tarasov and Potvin (2013) \\
\hline$[18]$ & $\mathrm{b}_{18}=\mathrm{dW}_{\alpha} / \mathrm{dW}_{\mathrm{e}}$ & $*_{\text {iv }}$ & Tarasov and Potvin (2013) \\
\hline [19] & $\mathrm{b}_{19}=\left(\mathrm{dW}_{\mathrm{f}}+\mathrm{dW}_{\mathrm{d}}\right) /\left(\mathrm{dW}_{\mathrm{ue}}+\mathrm{dW}_{\mathrm{d}}\right)$ & $*_{\mathrm{iv}}$ & Ai et al. (2016) \\
\hline$[20]$ & $\mathrm{b}_{20}=\mathrm{dW}_{\mathrm{x}} /\left(\mathrm{dW}_{\mathrm{ue}}+\mathrm{dW}_{\mathrm{d}}\right)$ & $*$ iv & Ai et al. (2016) \\
\hline [21] & $\mathrm{b}_{21}=\mathrm{E} / \mathrm{v}$ & $*_{\mathrm{V}}$ & Luan et al. (2014) \\
\hline$[22]$ & $\mathrm{b}_{22}=\left(\mathrm{E}_{\mathrm{n}}+\mathrm{v}_{\mathrm{n}}\right) / 2$ & $*_{\mathrm{V}}$ & Rickman et al. (2008) \\
\hline [23] & $\mathrm{b}_{23}=\mathrm{E} / \lambda$ & $*_{\mathrm{V}}$ & Chen et al. (2014) \\
\hline$[24]$ & $\mathrm{b}_{24}=(\lambda+2 \mathrm{G}) / \lambda$ & $*_{\mathrm{v}}$ & Guo et al. (2012) \\
\hline [25] & $\mathrm{b}_{25}=(3 \mathrm{~K}-5 \lambda) / \lambda$ & $*_{\mathrm{V}}$ & Huang et al. (2015) \\
\hline [26] & $\mathrm{b}_{26}=\mathrm{Ha} / \mathrm{Kc}$ & $*_{\mathrm{vi}}$ & Lawn and Marshall (1979) \\
\hline$[27]$ & $\mathrm{b}_{27}=\mathrm{Ha} \cdot \mathrm{E} / \mathrm{K}_{\mathrm{c}}{ }^{2}$ & $*_{\mathrm{vi}}$ & Quinn and Quinn (1997) \\
\hline [28] & $\mathrm{b}_{28}=\left(\mathrm{H}_{\mu}-\mathrm{H}_{\mathrm{m}}\right) / \mathrm{c}$ & $*_{\mathrm{vi}}$ & Hucka and Das (1974) \\
\hline [29] & $\mathrm{b}_{29}=\mathrm{q} \sigma_{\mathrm{c}}$ & $*_{\text {vii }}$ & Protodyakonov (1962) \\
\hline [30] & $\mathrm{b}_{30}=\mathrm{S}_{20}$ & *vii & Gong and Zhao (2007) \\
\hline [31] & $\mathrm{b}_{31}=\mathrm{F}_{\max } / \mathrm{P}$ & $*_{\text {viii }}$ & Yagiz (2009) \\
\hline [32] & $\mathrm{b}_{32}=\mathrm{P}_{\mathrm{dec}} / \mathrm{P}_{\mathrm{inc}}$ & $*_{\text {viii }}$ & Copur et al. (2003) \\
\hline [33] & $\mathrm{b}_{33}=\mathrm{K}_{\mathrm{b}}, \mathrm{K}_{\mathrm{s}} \mathrm{P} / \mathrm{h}^{2}=\mathrm{S}_{\mathrm{t}}-\mathrm{K}_{\mathrm{b}} \mathrm{P}$ & $*_{\mathrm{ix}}$ & Reichmuth (1968) \\
\hline [34] & $\mathrm{b}_{34}=\sigma_{\mathrm{c}} / \sigma_{\mathrm{c}-\mathrm{NC}}$ & $*_{\mathrm{X}}$ & Ingram and Urai (1999) \\
\hline [35] & $\mathrm{b}_{35}=\mathrm{OCR}^{\mathrm{b}}, \mathrm{OCR}=\sigma_{\mathrm{vmax}} / \sigma_{\mathrm{v}}$ & $*_{\mathrm{X}}$ & Nygård et al. (2006) \\
\hline [36] & $\mathrm{b}_{36}=\mathrm{W}_{\mathrm{q}} / \mathrm{W}_{\mathrm{t}}$ & *xi & Jarvie et al. (2007) \\
\hline [37] & $\mathrm{b}_{37}=\left(\mathrm{W}_{\mathrm{q}}+\mathrm{W}_{\mathrm{c}}\right) / \mathrm{W}_{\mathrm{t}}$ & *xi & Jin et al. (2014) \\
\hline [38] & $\mathrm{b}_{38}=-1.8748 \emptyset+0.9679$ & *xii & Jin et al. (2014) \\
\hline [39] & $\mathrm{b}_{39}=\sin \theta$ & $*_{\text {xiii }}$ & Hucka and Das (1974) \\
\hline [40] & $b_{40}=45^{\circ}+\theta / 2$ & *xiii & Hucka and Das (1974) \\
\hline
\end{tabular}

*Note that:

i. $\sigma \mathrm{c}$ and $\sigma \mathrm{t}$ are the uniaxial compression strength and tensile strength; $\tau \mathrm{p}$ and $\tau \mathrm{r}$ are the peak strength and residual strength; $\sigma c i$ and $\mathrm{K}$ are the initiation stress and initiation stress level,

ii. $\quad$ t and $\varepsilon r$ are the total strain and reversible strain at failure; cini is the strain at onset of fracture initiation (dilatancy); $\varepsilon p$ and $\varepsilon r$ are peak and residual strain; pc and $\varepsilon \mathrm{pf}$ are the plastic strain necessary for cohesion loss and frictional strengthening; $\varepsilon 11$ is the absolute irreversible longitudinal strain at failure,

iii. $\tau p$ and $\tau \mathrm{r}$ are the peak strength and residual strength, kac is the slope of the post-peak stress drop; $\sigma \mathrm{i}, \sigma \mathrm{p}$ and $\sigma \mathrm{r}$ are the initiation stress, peak strength and residual strength, $\varepsilon i, \varepsilon p$ and $\varepsilon r$ are initiation strain, peak strain and residual strain,

iv. Wr and Wt are the recoverable energy and total energy at failure; dWr, dWe and dW $\alpha$ are the post-peak rupture energy, the converted elastic energy and the released energy; $\mathrm{dWf}$ and $\mathrm{dWd}$ are the post-peak rupture energy and the pre-peak dissipation energy, and dWue and dWx are the unloading elastic energy and the extra energy required or the excess energy released,

v. E and $v$ are the Young's modulus and Poisson's ratio; En and vn are the normalized Young's modulus and Poisson's ratio; $\lambda$ and G are the first and second Lame parameters; $\mathrm{K}$ is the bulk modulus,

vi. Ha is the hardness, $\mathrm{Kc}$ is the fracture toughness, $\mathrm{E}$ is the elastic modulus; $\mathrm{H} \mu$ is the micro-indentation hardness, Hm is the macroindentation hardness, and $\mathrm{c}$ is a constant,

vii. $\mathrm{q}$ is the percentage of fines (below 28 mesh) obtained from Protodyakonov's impact test; S20 is the percentage of the fines less than $11.2 \mathrm{~mm}$,

viii. Fmax is the maximum applied force, $\mathrm{P}$ is the penetration depth at the maximum force; Pinc and Pdec are the average increment and decrement of forces,

ix. $\mathrm{Kb}$ is the relative brittleness index, $\mathrm{Ks}$ is the shape factor, $\mathrm{P}$ is the applied load at failure, $\mathrm{h}$ is the distance between loading points, and $\mathrm{St}$ is the tensile strength,

x. $\sigma \mathrm{c}-\mathrm{NC}$ is the uniaxial compression strength of a normally consolidated rock in non-overpressured areas; OCR is the over-consolidation ratio, $\mathrm{b}$ is the empirical constant, $\sigma \mathrm{vmax}$ and $\sigma \mathrm{v}$ are maximum effective vertical stress experienced and the current effective vertical stress,

xi. Wq is the weight of quartz, $\mathrm{Wc}$ is the carbonate mineral weight, and $\mathrm{Wt}$ is the total mineral weight,

xii. $\varnothing$ is the neutron porosity,

xiii. $\theta$ is the inner friction angle determined from Mohr's envelope at $\sigma v n=0$ 


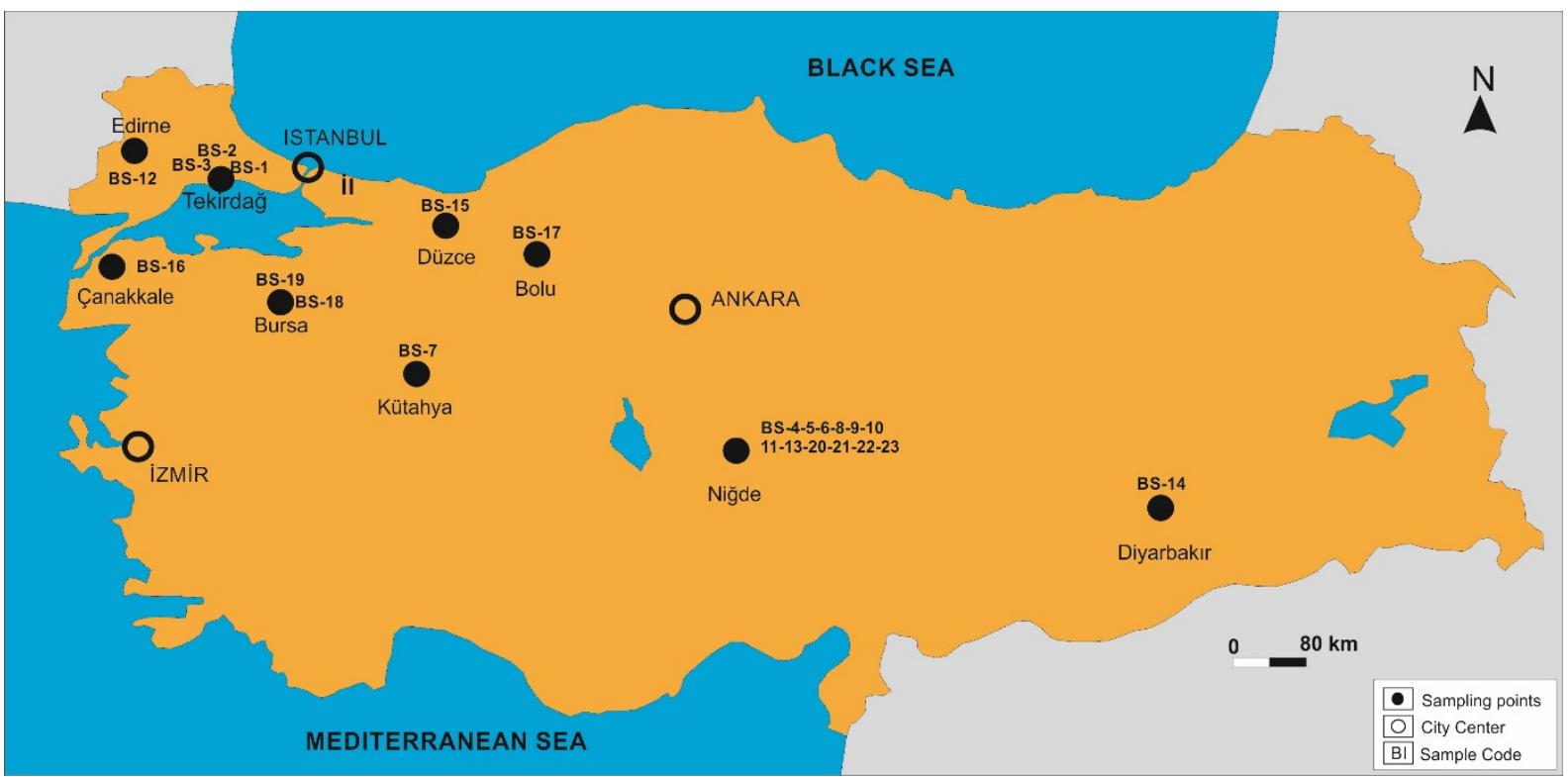

Fig. 1 Map showing the sampling locations of the basaltic rocks tested in the present work.

Although Zhang et al. (2021) have included 40 different brittleness index approach, Meng et al. (2021) have categorized 11 different groups of brittleness indices (a total of 80 brittleness indices) in their review (Table A1 from Meng et al. (2021)). Mikaeil et. al. (2013) founded a mathematical correlation between production rate in ornamental stones sawing and rock brittleness index. According to Mikaeil et. al. (2013), the production rate decreases with the increase in BI and this fact can be used as a parameter for predicting the production rate for all type of ornamental stones. Some brittleness index definitions derived from stress-strain curves were introduced and used in the literatures (Aubertin et al., 1994; Ribacchi, 2000; Hajiabdolmajid and Kaiser, 2003; Tarasov and Potvin, 2013; Meng et al., 2015, Xia et al., 2017; Zhou et al., 2018). Also some researches founded relationships between brittleness and drillability, borability, the specific energy in rock cutting, shore hardness (Kahraman, 2002; Altindag 2000, 2002, 2003; Kahraman and Altindag, 2004; Altindag and Guney, 2010). According to Aligholi (2017), there is a systematic trend between dry density, P-wave velocity, rebound hardness and brittleness value of the studied igneous rocks with medium hardness. The brittleness characteristics of rocks affect their mechanical performance, drillability, cuttability, and machine performance (Aligholi, 2017).

The aim of this study is to evaluate brittleness properties of basaltic rock samples collected from different regions of Turkey and to predict brittleness behavior based on chemical parameter (CP) in terms of their major oxide element contents. The brittleness indices, i.e. B1, B2 and B3 were calculated by employing the uniaxial compressive strength (UCS) and Brazilian tensile strength (BTS) of the rocks. In addition, a new chemical parameter (CP) was proposed to be in relationship with these abovementioned brittleness index values, i.e. B1, B2, and $\mathrm{B} 3$. Corresponding calculation of $\mathrm{CP}$ is performed with the consideration of chemical properties $\left(\mathrm{Al}_{2} \mathrm{O}_{3}\right.$, $\mathrm{Fe}_{2} \mathrm{O}_{3}, \mathrm{MgO}, \mathrm{SiO}_{2}$ values) of the rocks. The obtained results were compared with simple regression method.

\section{MATERIAL AND METHOD}

\subsection{PETROGRAPHIC AND CHEMICAL PROPERTIES}

Basaltic rocks have different geomechanical properties such as mineral content, chemical composition, and geomechanical properties. Therefore, in this study samples were collected different regions of Turkey (Fig. 1). In addition to the samples collected, some samples are adapted from the study of Korkanç (2003) in order to provide sample variety. After collection of basaltic rock samples, petrographic studies were carried out. Thin sections were prepared to determine the petrographic characteristics of different types of basalt samples. Detailed investigations on thin section samples were examined and photographed by using the LEICA DM $750 \mathrm{P}$ model polarizing microscope in the context of petrographical observations. With the help of abovementioned petrographical analysis, microstructures of each sample were defined. Corresponding description of thin sections, petrographic classification, rock name, mineral content and texture are tabulated in Table 2. In this context, mineralogical - petrographical analysis along with the chemical composition determination of the basaltic rocks samples were performed. In order to specify physical and mechanical properties of rocks; water absorption, unit weight, porosity, uniaxial compressive strength (UCS), Brazilian tensile strength (BTS), point load strength (Is ${ }_{50}$ ) and $\mathrm{P}$ wave velocity experiments were carried out. The corresponding sample number, location, mineral content, texture, 
Table 2 Sample no, location and petrographic classification of the studied samples.

\begin{tabular}{|c|c|c|c|c|c|c|}
\hline $\begin{array}{c}\text { Sample } \\
\text { No }\end{array}$ & $\begin{array}{l}\text { Sampling } \\
\text { location }\end{array}$ & $\begin{array}{l}\text { Mineral } \\
\text { content } \\
\text { and } \\
\text { matrix }\end{array}$ & Texture & $\begin{array}{c}\text { Weathering/ } \\
\text { Hydrotermal } \\
\text { Alteration }\end{array}$ & $\begin{array}{l}\text { Rock } \\
\text { Name }\end{array}$ & Thin section image \\
\hline & Tekirdağ & $\begin{array}{l}\text { Ol, Px, Pl, } \\
\text { OM, Gl }\end{array}$ & $\begin{array}{l}\text { Hypocrystalline } \\
\text { porphyritic }\end{array}$ & $\begin{array}{l}\text { Chloritization, } \\
\text { silification }\end{array}$ & $\begin{array}{l}\text { Olivine } \\
\text { Basalt }\end{array}$ & \\
\hline \multicolumn{7}{|l|}{ BS-1 } \\
\hline & Tekirdağ & $\begin{array}{l}\text { Ol, Px, Pl, } \\
\text { OM }\end{array}$ & $\begin{array}{l}\text { Hypocrystalline } \\
\text { porphyritic }\end{array}$ & Sericitization & $\begin{array}{l}\text { Olivine } \\
\text { Basalt }\end{array}$ & \\
\hline \multicolumn{7}{|l|}{ BS-2 } \\
\hline & Tekirdağ & $\begin{array}{l}\mathrm{Px}, \mathrm{Pl}, \mathrm{Ol}, \\
\mathrm{OM}\end{array}$ & $\begin{array}{l}\text { Holocrystalline } \\
\text { porphyritic }\end{array}$ & Silification & Basalt & \\
\hline \multicolumn{7}{|l|}{ BS-3 } \\
\hline & Niğde & $\begin{array}{l}\text { CPx, Ol, } \\
\mathrm{Pl}\end{array}$ & Pylotacsitic & - & Basalt & \\
\hline & Niğde & $\begin{array}{l}\text { Ol, Px, Pl, } \\
\text { OM }\end{array}$ & Pylotacsitic & - & Basalt & \\
\hline & Niğde & $\begin{array}{l}\text { Microlitic } \\
\text { P1, O1, } \\
\text { OM }\end{array}$ & Pylotacsitic & - & $\begin{array}{l}\text { Olivine } \\
\text { Basalt }\end{array}$ & \\
\hline & Kütahya & $\begin{array}{l}\mathrm{Px}, \mathrm{Pl}, \mathrm{Ol}, \\
\mathrm{OM}\end{array}$ & $\begin{array}{l}\text { Holocrystalline } \\
\text { porphyritic }\end{array}$ & $\begin{array}{l}\text { Carbonation, } \\
\text { argillisation, } \\
\text { talkization }\end{array}$ & $\begin{array}{l}\text { Olivine } \\
\text { Basalt }\end{array}$ & \\
\hline \multicolumn{7}{|l|}{ BS-7 } \\
\hline
\end{tabular}




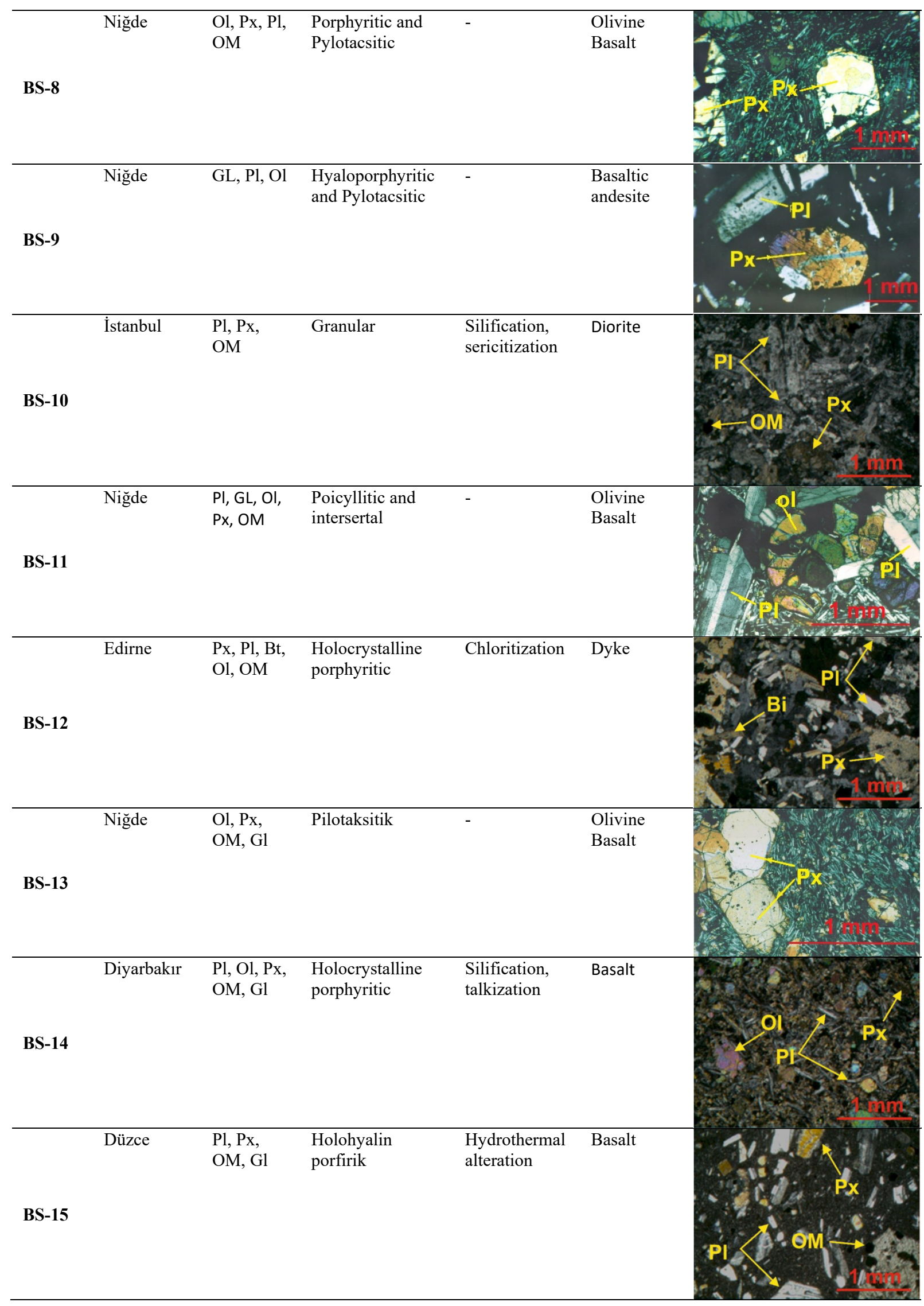




$\begin{array}{lllll}\text { Çanakkale } & \text { Pl, Px, } & \begin{array}{l}\text { Hypocrystalline } \\ \text { porphyritic }\end{array} & \begin{array}{l}\text { Hydrothermal } \\ \text { alteration, } \\ \text { carbonation }\end{array} & \text { Basalt } \\ & & & \end{array}$

BS-16

$\begin{array}{lllll}\text { Bolu } & \text { Pl, Px, } & \begin{array}{l}\text { Hypocrystalline } \\ \text { porphyritic }\end{array} & \begin{array}{l}\text { Hydrothermal } \\ \text { alteration }\end{array} & \text { Basalt } \\ & \text { OM, Gl } & \end{array}$

BS-17

$\begin{array}{lllll}\text { Bursa } & \text { Pl, Px, } & \begin{array}{l}\text { Hypocrystalline } \\ \text { porphyritic }\end{array} & \begin{array}{l}\text { Hydrothermal } \\ \text { alteration }\end{array} & \text { Basalt } \\ & \text { OM, Gl } & \end{array}$

BS-18

$\begin{array}{lllll}\text { Bursa } & \begin{array}{l}\mathrm{Pl}, \mathrm{Px}, \mathrm{Ol}, \\ \mathrm{OM}, \mathrm{Gl}\end{array} & \begin{array}{l}\text { Hypocrystalline } \\ \text { porphyritic }\end{array} & \begin{array}{l}\text { Hydrothermal } \\ \text { alteration, } \\ \text { carbonation }\end{array} & \text { Basalt } \\ & & & \end{array}$

BS-19

\begin{tabular}{|c|c|c|}
\hline ğde & $\begin{array}{l}\text { Ol, Px, } \\
\text { Pl }\end{array}$ & Pylotacsitic \\
\hline
\end{tabular}

BS-20

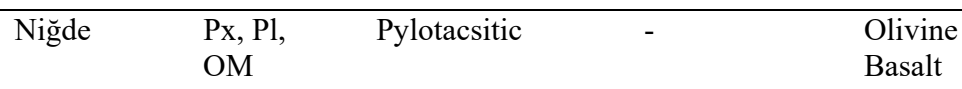

BS-21

$\begin{array}{lllll}\text { Niğde } & \text { Pl, Gl, Ol } & \text { Pylotacsitic } & - & \text { Basalt }\end{array}$

BS-22

$\begin{array}{lllll}\text { Niğde } & \text { Px, Ol, Pl, } & \text { Pylotacsitic } & - & \text { Basalt }\end{array}$

"BS-23

(CPx: Clinopyroxene, Px: Pyroxene, Pl: Plagioclase, Ol: Olivine,. OM: Opaque Mineral , Bt: Biotite, Volcanic Glass: Gl)

* Acording to LeBas et al. (1991). 
Table 3 Chemical and mineralogical properties of rock samples.

\begin{tabular}{|c|c|c|c|c|c|c|c|c|c|c|c|c|c|}
\hline $\begin{array}{c}\text { Sample } \\
\text { No } \\
\end{array}$ & $\begin{array}{l}\mathrm{SiO}_{2} \\
(\%)\end{array}$ & $\begin{array}{c}\mathrm{Al}_{2} \mathrm{O}_{3} \\
(\%)\end{array}$ & $\begin{array}{c}\mathrm{Fe}_{2} \mathrm{O}_{3} \\
(\%)\end{array}$ & $\begin{array}{l}\mathrm{CaO} \\
(\%)\end{array}$ & $\begin{array}{c}\mathrm{MgO} \\
(\%)\end{array}$ & $\begin{array}{l}\mathrm{K}_{2} \mathrm{O} \\
(\%)\end{array}$ & $\begin{array}{c}\mathrm{Na}_{2} \mathrm{O} \\
(\%)\end{array}$ & $\begin{array}{l}\text { LOI } \\
(\%)\end{array}$ & $\begin{array}{l}\text { Px } \\
(\%)\end{array}$ & $\begin{array}{c}\text { PI } \\
(\%)\end{array}$ & $\begin{array}{c}\text { OI } \\
(\%)\end{array}$ & $\begin{array}{l}\text { OM } \\
(\%)\end{array}$ & $\begin{array}{c}\text { Matrix } \\
(\%)\end{array}$ \\
\hline BS-1 & 46.14 & 13.42 & 10.33 & 8.89 & 12.18 & 2.12 & 3.15 & 1.02 & 9.8 & 7.8 & 18.2 & 2.3 & 64.9 \\
\hline BS-2 & 45.22 & 13.17 & 10.32 & 9.44 & 12.36 & 1.72 & 3.17 & 3.66 & 9.1 & 13.1 & 19.5 & 0.6 & 60.7 \\
\hline BS-3 & 46.17 & 13.71 & 10.44 & 9.63 & 11.52 & 2.44 & 4.88 & 0.71 & 9.3 & 2.1 & 14.2 & 0.4 & 74.4 \\
\hline BS-4 & 60.92 & 16.99 & 5.50 & 7.12 & 2.67 & 1.21 & 3.3 & 1.2 & 9 & 0 & 4.1 & 2 & 85.6 \\
\hline BS-5 & 60.97 & 17.01 & 5.48 & 7.09 & 2.96 & 1.21 & 3.27 & 0.6 & 8.2 & 1.5 & 3.1 & 4.6 & 82.8 \\
\hline BS-6 & 49.06 & 16.65 & 9.16 & 9.52 & 7.96 & 0.89 & 3.41 & 0.8 & 10.7 & 0 & 12.2 & 4.9 & 73.2 \\
\hline BS-7 & 52.01 & 18.12 & 6.05 & 9.02 & 3.14 & 2.51 & 3.43 & 5.78 & 18.7 & 6.3 & 8.5 & 0.7 & 67.8 \\
\hline BS-8 & 48.36 & 16.05 & 8.49 & 10.69 & 9.12 & 0.67 & 3.34 & 1 & 10.3 & 0 & 10.1 & 6 & 74.3 \\
\hline BS-9 & 54.36 & 20.37 & 7.04 & 8.96 & 2.67 & 1.81 & 2.97 & 0.6 & 0.3 & 40.1 & 7.8 & 1.3 & 51.2 \\
\hline BS-10 & 60.15 & 15.13 & 5.49 & 4.98 & 2.86 & 0.56 & 2.97 & 4.5 & 17.6 & 22.2 & - & 1.5 & 58.7 \\
\hline BS-11 & 49.69 & 15.48 & 8.21 & 11.02 & 9.14 & 1 & 3.05 & 0.4 & 7.8 & 1 & 15.8 & 3.8 & 73.6 \\
\hline BS-12 & 49.72 & 12.21 & 7.94 & 7.88 & 12.71 & 5.63 & 2.58 & 1.72 & 26 & 12.4 & 12.1 & 0.4 & 49.1 \\
\hline BS-13 & 49.17 & 15.91 & 8.74 & 10.7 & 9.31 & 0.68 & 3.33 & 0.1 & 9.5 & 0 & 16.8 & 5.8 & 68.3 \\
\hline BS-14 & 47.56 & 14.6 & 14.31 & 10.97 & 6.45 & 1.18 & 3.04 & 0.36 & 10.9 & 22.2 & 14.8 & 0.2 & 51.8 \\
\hline BS-15 & 58.12 & 18.16 & 6.72 & 6.51 & 3.14 & 1.28 & 3.49 & 1.05 & 13.4 & 27.3 & - & 0.8 & 58.5 \\
\hline BS-16 & 56.52 & 18.43 & 6.09 & 6.78 & 3.27 & 3.33 & 2.97 & 1.98 & 12.7 & 31.1 & - & 0.3 & 57.1 \\
\hline BS-17 & 58.7 & 16.99 & 7.91 & 5.64 & 3.26 & 1.7 & 4.16 & 1.59 & 16.4 & 27.9 & - & 1.3 & 54.2 \\
\hline BS-18 & 56.98 & 17.82 & 7.05 & 6.89 & 3.88 & 2.02 & 3.41 & 1.52 & 22.3 & 28.1 & - & 0.5 & 48.8 \\
\hline BS-19 & 54.28 & 19.93 & 8.96 & 7.70 & 2.76 & 1.12 & 3.51 & 2.09 & 4.8 & 30.8 & 7.4 & 1.1 & 57.6 \\
\hline BS-20 & 51.19 & 17.14 & 8.13 & 9.12 & 6.5 & 1.61 & 3.83 & 0.1 & 5.8 & 0.8 & 8 & 3.5 & 82 \\
\hline BS-21 & 51.07 & 17.02 & 8.06 & 9.08 & 6.61 & 1.63 & 3.83 & 0.2 & 10.9 & 2.3 & 10.8 & 5.6 & 72.1 \\
\hline BS-22 & 60.89 & 16.9 & 5.41 & 7.17 & 3.23 & 1.28 & 3.23 & 0.7 & 0.1 & 0 & 5.2 & 2.8 & 90.4 \\
\hline BS-23 & 60.97 & 16.79 & 5.40 & 7.29 & 2.98 & 1.18 & 3.26 & 0.9 & 7.6 & 0 & 2.6 & 1.8 & 88.4 \\
\hline
\end{tabular}

(LOI: Loss on ignition, Px: Pyroxene, Pl: Plagioclase, Ol: Olivine, OM: Opaque Mineral)

rock name and thin section images of the samples investigated in this study are tabulated in Table 2 (Thin section images of the samples from Niğde region, i.e. samples of BS 4-5-6-8-9-10-11-13-20-21-22-23 are adapted from the studies of Korkanç (2003), Korkanç and Tuğrul (2004), Korkanç and Tuğrul (2005). In terms of the classification of basaltic rocks, LeBas et al. (1991) classification system was taken into consideration. According to the system abovementioned, rock samples were described as olivine basalt, basalt, basaltic andesite, diorite and dyke. The most common minerals are olivine, pyroxene, plagioclase, opaque mineral and volcanic glass. Right after the completion of petrographic studies, major element oxides analysis was calculated on the granulated rock samples and obtained results are provided in Table 3 . In terms of chemical analysis, the ICP (Inductively Coupled Plasma) method was applied to powder samples $(<200 \mu \mathrm{m})$ (obtained by grinding at laboratory scale mills) to determine the major element oxides contents of basalts with different facies.

Basalt materials are classified according to their $\mathrm{SiO}_{2}$ content as alkaline basalts (up to $42 \% \mathrm{SiO}_{2}$ ), mildly acidic basalts (43 to $46 \% \mathrm{SiO}_{2}$ ) and acidic basalts (over $46 \% \mathrm{SiO}_{2}$ ). Basaltic rocks which has $\mathrm{SiO}_{2}$ content above $46 \%$ (acidic basalt) with constant composition show ability to melt without solid residue, appropriate melt viscosity for fiber formation and ability to solidify in a glassy phase without noticeable crystallization (Park et al., 1999; Militky and Kovacic, 1996). Almost all samples studied have volcanic glass in their mineral content and matrix. Studied basaltic rocks samples consist of pyroxene, plagioclase and olivine minerals.As shown in Table 3, $\mathrm{SiO}_{2}$ contents of studied samples are in a range between $45.22 \%$ and $60.97 \%$.

\subsection{PHYSICAL AND MECHANICAL PROPERTIES}

Block samples with an average size of $30 \times 30 \times 30 \mathrm{~cm}$ were collected to be used in laboratory experiments. Determination of physical properties (unit weight, porosity, water absorption and $\mathrm{P}$ wave velocity) for each corresponding basalt sample were carried out according to the method suggested by ISRM (2007). The mechanical properties of the samples were determined according to ASTM (2001a, 2010). Mechanical properties such as uniaxial compressive strength (UCS), Brazilian tensile strength (BTS) and Point load strength index (Is ${ }_{50}$ ) were determined on the core samples. In terms of physical properties determination, cylindrical core samples with a length / diameter ratio between 2.0-2.5 were prepared in accordance with ISRM (2007) standards. Experiments were performed on basalt samples with $54.7 \mathrm{~mm}(\mathrm{NX}), 42 \mathrm{~mm}(\mathrm{BX})$ and $36.5 \mathrm{~mm}(\mathrm{BQ})$ diameter. In this context, a total of 23 samples were tested and each test results are tabulated in Table 4. The physical and mechanical properties of the studied basaltic rocks were also compared with each other (Figs. 2 and 3). 
Table 3 Chemical and mineralogical properties of rock samples.

\begin{tabular}{|c|c|c|c|c|c|c|c|c|c|c|c|c|c|}
\hline $\begin{array}{c}\text { Sample } \\
\text { No }\end{array}$ & $\begin{array}{l}\mathrm{SiO}_{2} \\
(\%)\end{array}$ & $\begin{array}{c}\mathrm{Al}_{2} \mathrm{O}_{3} \\
(\%)\end{array}$ & $\begin{array}{c}\mathrm{Fe}_{2} \mathrm{O}_{3} \\
(\%)\end{array}$ & $\begin{array}{c}\mathrm{CaO} \\
(\%)\end{array}$ & $\begin{array}{c}\text { MgO } \\
(\%)\end{array}$ & $\begin{array}{l}\mathrm{K}_{2} \mathrm{O} \\
(\%)\end{array}$ & $\begin{array}{c}\mathrm{Na}_{2} \mathrm{O} \\
(\%)\end{array}$ & $\begin{array}{l}\text { LOI } \\
(\%)\end{array}$ & $\begin{array}{c}\text { Px } \\
(\%)\end{array}$ & $\begin{array}{c}\text { PI } \\
(\%)\end{array}$ & $\begin{array}{c}\text { Ol } \\
(\%)\end{array}$ & $\begin{array}{l}\text { OM } \\
(\%)\end{array}$ & $\begin{array}{c}\text { Matrix } \\
(\%)\end{array}$ \\
\hline BS-1 & 46.14 & 13.42 & 10.33 & 8.89 & 12.18 & 2.12 & 3.15 & 1.02 & 9.8 & 7.8 & 18.2 & 2.3 & 64.9 \\
\hline BS-2 & 45.22 & 13.17 & 10.32 & 9.44 & 12.36 & 1.72 & 3.17 & 3.66 & 9.1 & 13.1 & 19.5 & 0.6 & 50.7 \\
\hline BS-3 & 46.17 & 13.71 & 10.44 & 9.63 & 11.52 & 2.44 & 4.88 & 0.71 & 9.3 & 2.1 & 14.2 & 0.4 & 74.4 \\
\hline BS-4 & 60.92 & 16.99 & 5.50 & 7.12 & 2.67 & 1.21 & 3.3 & 1.2 & 9 & 0 & 4.1 & 2 & 85.6 \\
\hline BS-5 & 60.97 & 17.01 & 5.48 & 7.09 & 2.96 & 1.21 & 3.27 & 0.6 & 8.2 & 1.5 & 3.1 & 4.6 & 82.8 \\
\hline BS-6 & 49.06 & 16.65 & 9.16 & 9.52 & 7.96 & 0.89 & 3.41 & 0.8 & 10.7 & 0 & 12.2 & 4.9 & 73.2 \\
\hline BS-7 & 52.01 & 18.12 & 6.05 & 9.02 & 3.14 & 2.51 & 3.43 & 5.78 & 18.7 & 6.3 & 8.5 & 0.7 & 67.8 \\
\hline BS-8 & 48.36 & 16.05 & 8.49 & 10.69 & 9.12 & 0.67 & 3.34 & 1 & 10.3 & 0 & 10.1 & 6 & 74.3 \\
\hline BS-9 & 54.36 & 20.37 & 7.04 & 8.96 & 2.67 & 1.81 & 2.97 & 0.6 & 0.3 & 40.1 & 7.8 & 1.3 & 51.2 \\
\hline BS-10 & 60.15 & 15.13 & 5.49 & 4.98 & 2.86 & 0.56 & 2.97 & 4.5 & 17.6 & 22.2 & - & 1.5 & 58.7 \\
\hline BS-11 & 49.69 & 15.48 & 8.21 & 11.02 & 9.14 & 1 & 3.05 & 0.4 & 7.8 & 1 & 15.8 & 3.8 & 73.6 \\
\hline BS-12 & 49.72 & 12.21 & 7.94 & 7.88 & 12.71 & 5.63 & 2.58 & 1.72 & 26 & 12.4 & 12.1 & 0.4 & 49.1 \\
\hline BS-13 & 49.17 & 15.91 & 8.74 & 10.7 & 9.31 & 0.68 & 3.33 & 0.1 & 9.5 & 0 & 16.8 & 5.8 & 68.3 \\
\hline BS-14 & 47.56 & 14.6 & 14.31 & 10.97 & 6.45 & 1.18 & 3.04 & 0.36 & 10.9 & 22.2 & 14.8 & 0.2 & 51.8 \\
\hline BS-15 & 58.12 & 18.16 & 6.72 & 6.51 & 3.14 & 1.28 & 3.49 & 1.05 & 13.4 & 27.3 & - & 0.8 & 58.5 \\
\hline BS-16 & 56.52 & 18.43 & 6.09 & 6.78 & 3.27 & 3.33 & 2.97 & 1.98 & 12.7 & 31.1 & - & 0.3 & 57.1 \\
\hline BS-17 & 58.7 & 16.99 & 7.91 & 5.64 & 3.26 & 1.7 & 4.16 & 1.59 & 16.4 & 27.9 & - & 1.3 & 54.2 \\
\hline BS-18 & 56.98 & 17.82 & 7.05 & 6.89 & 3.88 & 2.02 & 3.41 & 1.52 & 22.3 & 28.1 & - & 0.5 & 48.8 \\
\hline BS-19 & 54.28 & 19.93 & 8.96 & 7.7 & 2.76 & 1.12 & 3.51 & 2.09 & 4.8 & 30.8 & 7.4 & 1.1 & 57.6 \\
\hline BS-20 & 51.19 & 17.14 & 8.13 & 9.12 & 6.5 & 1.61 & 3.83 & 0.1 & 5.8 & 0.8 & 8 & 3.5 & 82 \\
\hline BS-21 & 51.07 & 17.02 & 8.06 & 9.08 & 6.61 & 1.63 & 3.83 & 0.2 & 10.9 & 2.3 & 10.8 & 5.6 & 72.1 \\
\hline BS-22 & 60.89 & 16.9 & 5.41 & 7.17 & 3.23 & 1.28 & 3.23 & 0.7 & 0.1 & 0 & 5.2 & 2.8 & 90.4 \\
\hline BS-23 & 60.97 & 16.79 & 5.40 & 7.29 & 2.98 & 1.18 & 3.26 & 0.9 & 7.6 & 0 & 2.6 & 1.8 & 88.4 \\
\hline
\end{tabular}

Table 4 Geomechanical properties of rock samples.

\begin{tabular}{|c|c|c|c|c|c|c|c|}
\hline $\begin{array}{l}\text { Sample } \\
\text { No }\end{array}$ & $\begin{array}{c}\text { Unit } \\
\text { weight } \\
\left(\mathrm{kN} / \mathrm{m}^{3}\right)\end{array}$ & $\begin{array}{c}\text { Porosity } \\
(\%)\end{array}$ & $\begin{array}{c}\text { Water } \\
\text { abs. } \\
(\%)\end{array}$ & $\begin{array}{l}\text { P-wave } \\
\text { velocity } \\
\text { (m/sec) }\end{array}$ & $\begin{array}{c}\text { Point } \\
\text { load strength } \\
\text { index } \\
\text { (MPa) }\end{array}$ & $\begin{array}{c}\text { Uniaxial } \\
\text { compressive } \\
\text { strength } \\
\text { (MPa) }\end{array}$ & $\begin{array}{c}\text { Brazilian } \\
\text { tensile } \\
\text { strength } \\
\text { (MPa) }\end{array}$ \\
\hline BS-1 & 28.10 & 1.81 & 0.72 & 6988 & 19.88 & 256.00 & 29.00 \\
\hline BS-2 & 28.78 & 0.94 & 0.26 & 6974 & 18.24 & 277.00 & 26.00 \\
\hline BS-3 & 28.53 & 0.55 & 0.21 & 6523 & 18.01 & 241.00 & 33.00 \\
\hline BS-4 & 25.48 & 5.63 & 0.93 & 4959 & 7.11 & 166.70 & 17.00 \\
\hline BS-5 & 25.55 & 6.34 & 0.82 & 4907 & 8.90 & 175.50 & 17.00 \\
\hline BS-6 & 26.64 & 8.01 & 0.90 & 5873 & 16.56 & 188.80 & 24.00 \\
\hline BS-7 & 27.18 & 1.27 & 0.49 & 5627 & 12.89 & 186.00 & 24.00 \\
\hline BS-8 & 27.18 & 3.95 & 0.82 & 6175 & 17.91 & 195.30 & 29.00 \\
\hline BS-9 & 27.52 & 2.63 & 0.57 & 5401 & 11.19 & 173.90 & 21.00 \\
\hline BS-10 & 26.40 & 0.80 & 0.45 & 5236 & 7.85 & 191.00 & 20.00 \\
\hline BS-11 & 28.34 & 2.40 & 0.62 & 5817 & 15.37 & 177.90 & 23.00 \\
\hline BS-12 & 27.77 & 1.88 & 0.88 & 5867 & 14.82 & 175.00 & 19.00 \\
\hline BS-13 & 27.51 & 9.75 & 0.71 & 5957 & 16.67 & 232.30 & 27.00 \\
\hline BS-14 & 28.51 & 0.83 & 0.64 & 6328 & 18.74 & 268.00 & 26.00 \\
\hline BS-15 & 27.14 & 0.21 & 0.13 & 5306 & 8.23 & 190.00 & 21.00 \\
\hline BS-16 & 26.30 & 0.40 & 0.16 & 5502 & 9.99 & 188.00 & 18.00 \\
\hline BS-17 & 26.68 & 0.71 & 0.49 & 5368 & 7.64 & 194.00 & 19.00 \\
\hline BS-18 & 26.65 & 0.42 & 0.21 & 5564 & 7.68 & 173.00 & 20.00 \\
\hline BS-19 & 26.63 & 1.28 & 0.61 & 5428 & 10.52 & 186.00 & 19.00 \\
\hline BS-20 & 26.16 & 10.01 & 0.80 & 5694 & 13.93 & 182.60 & 19.00 \\
\hline BS-21 & 26.87 & 6.41 & 0.82 & 5718 & 14.42 & 167.50 & 22.00 \\
\hline BS-22 & 25.21 & 6.33 & 1.16 & 4737 & 7.10 & 165.30 & 19.00 \\
\hline BS-23 & 25.83 & 3.68 & 0.71 & 5219 & 7.22 & 136.10 & 16.00 \\
\hline
\end{tabular}




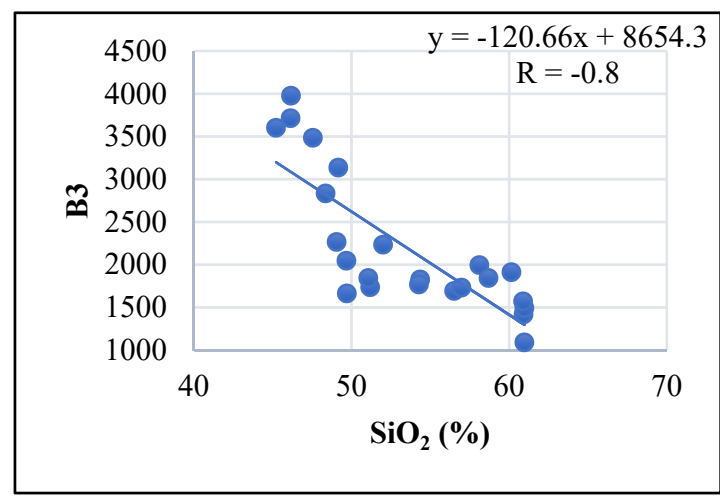

(a)

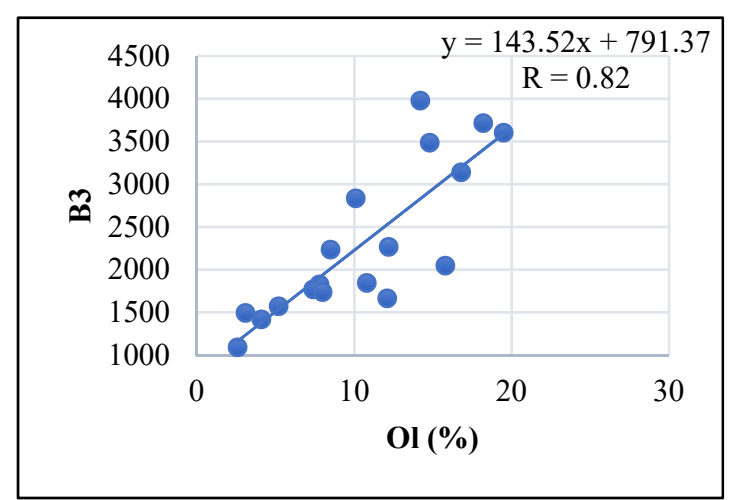

(d)

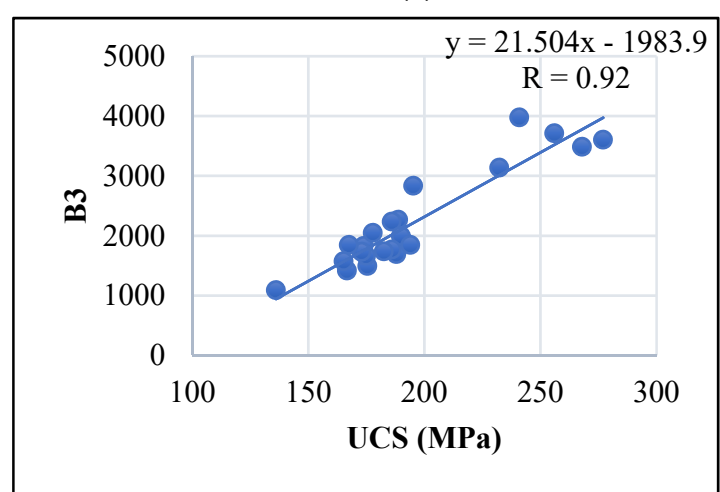

(g)

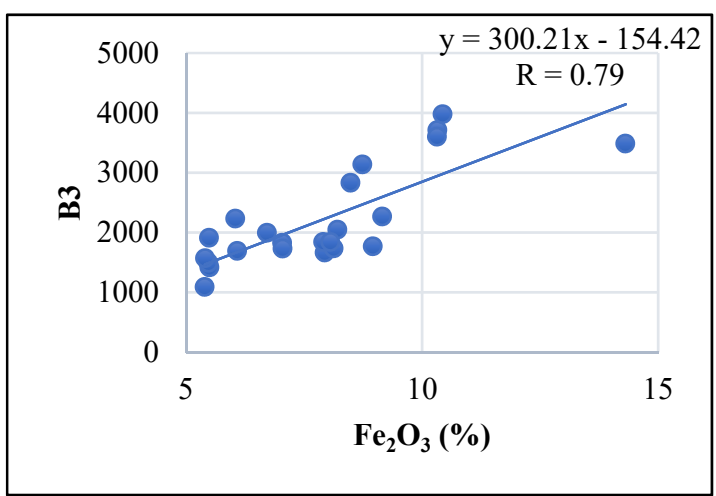

(b)

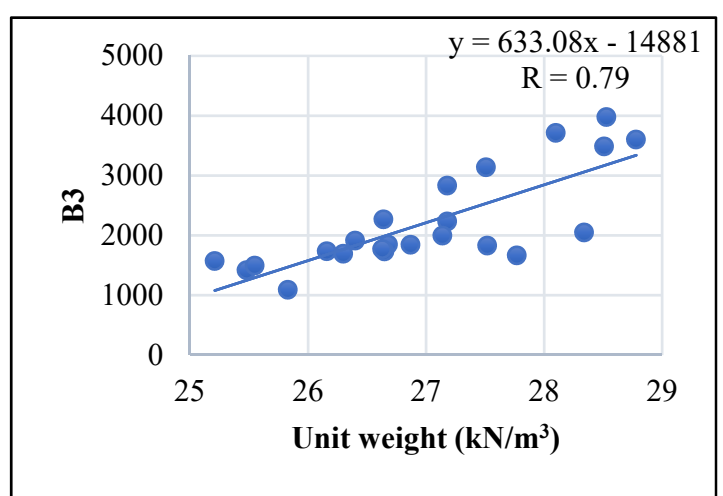

(e)

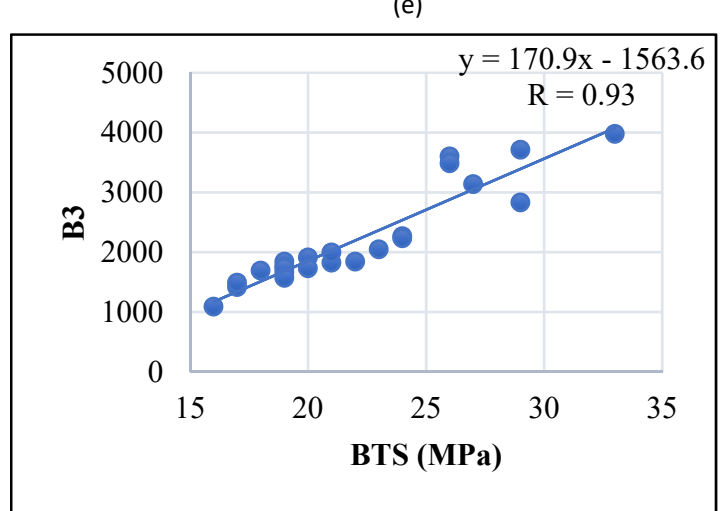

()h

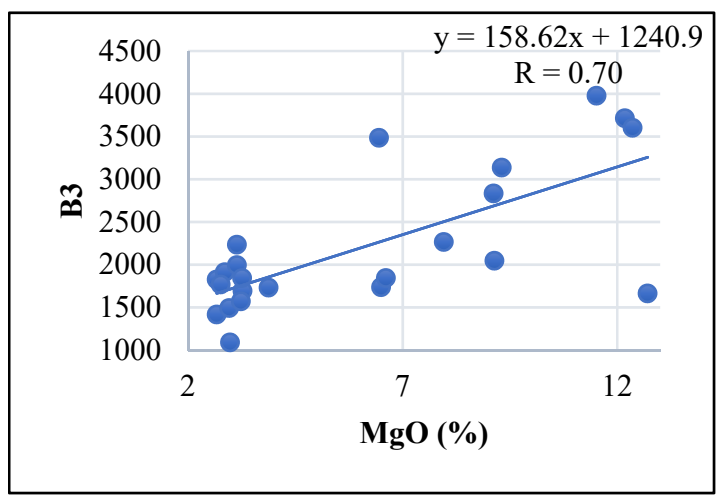

(c)
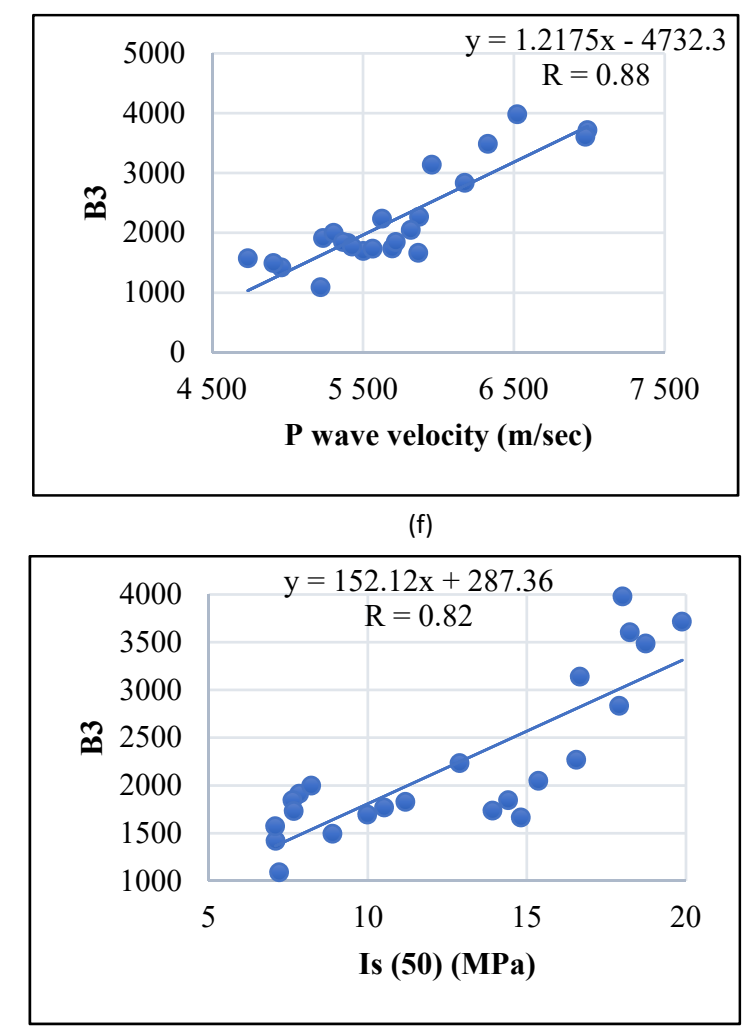

(i)

Fig. 2 Correlation between brittlenes indices (B3) and $\mathrm{SiO}_{2}(\mathrm{a})-\mathrm{Fe}_{2} \mathrm{O}_{3}$ (b) - $\mathrm{MgO}$ (c) - olivine (d) - unit weight (e) - P wave velocity (f) - UCS (g) - BTS (h) - Is (50) (i) content. 

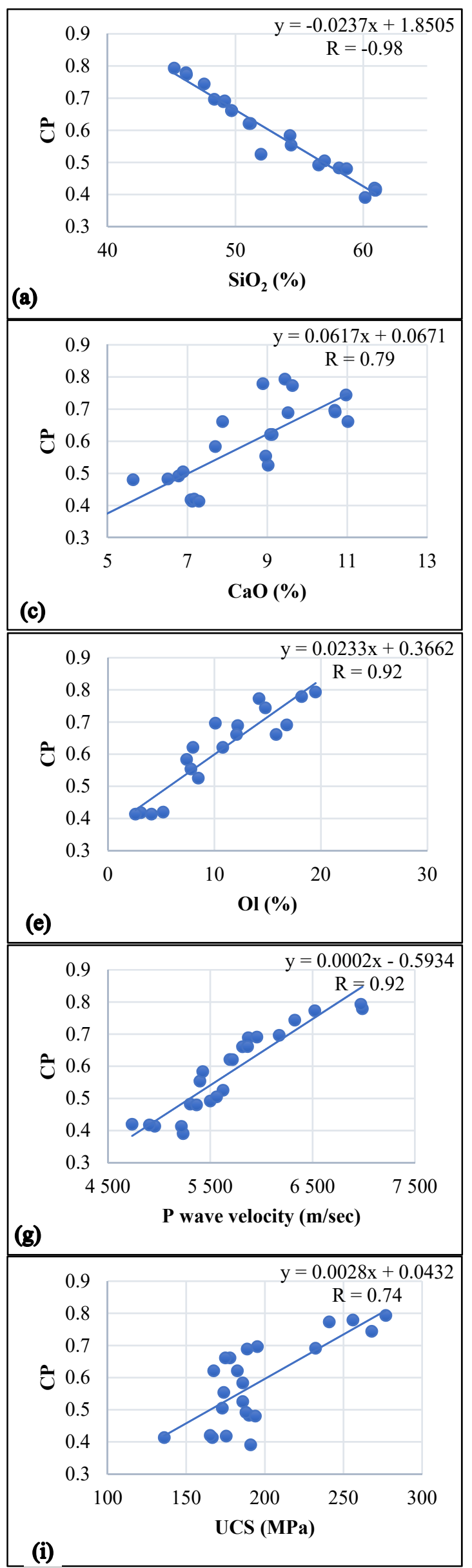

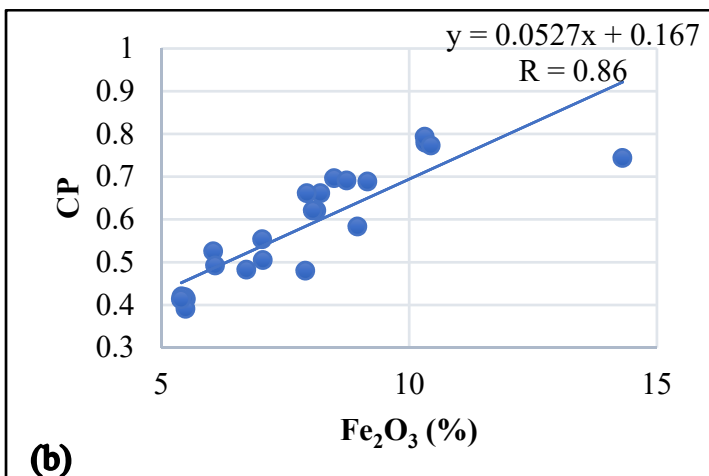

(b)
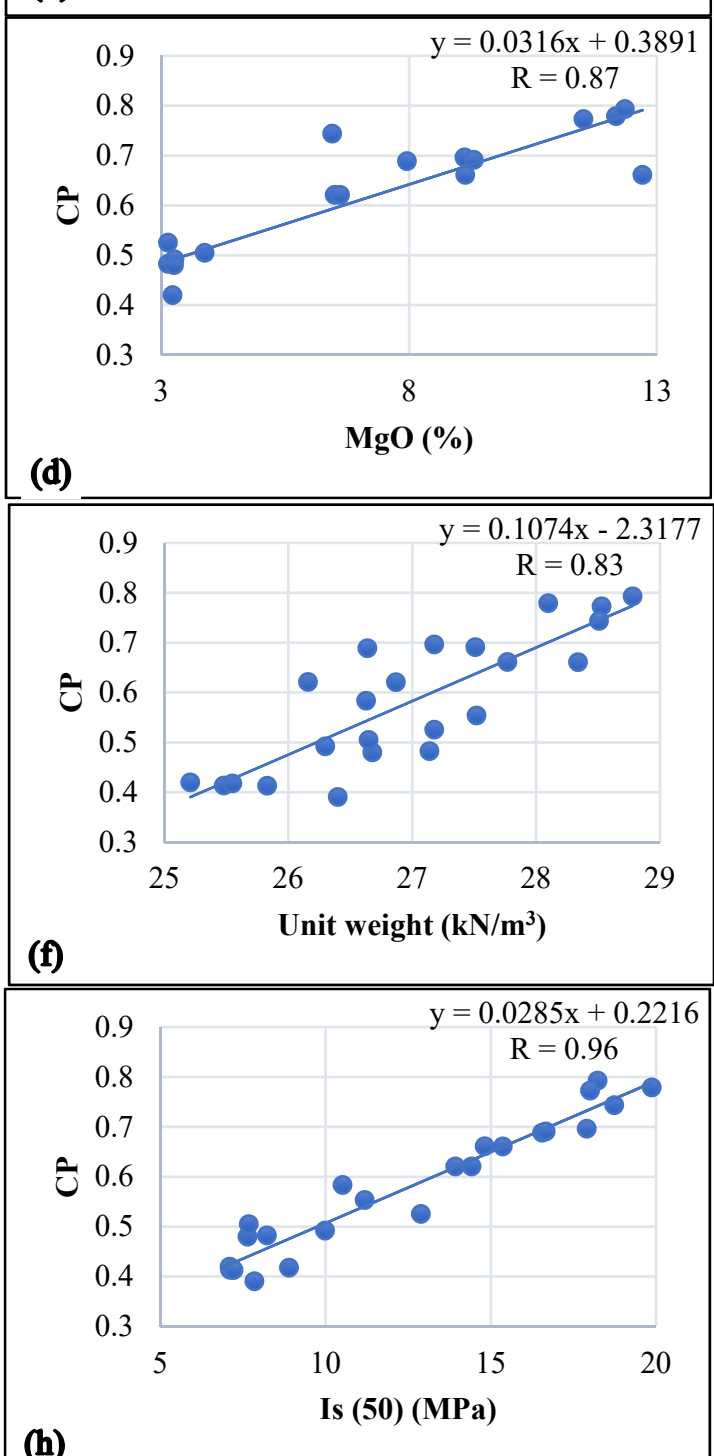

(h)

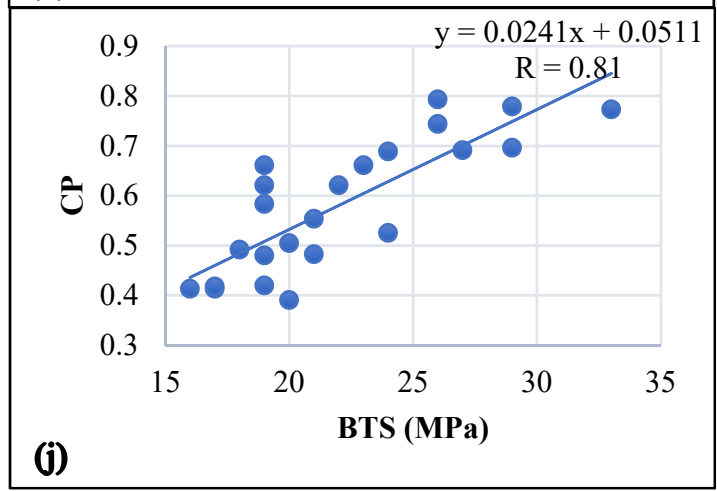

Fig. 3 Correlation between chemical parameter (CP) and $\mathrm{SiO}_{2}(\mathrm{a})-\mathrm{Fe}_{2} \mathrm{O}_{3}$ (b) $-\mathrm{CaO}$ (c) $-\mathrm{MgO}$ (d) olivine (e) - unit weight (f) - P wave velocity (g) - Is (50) (h) - UCS (i) - BTS (j). 
Table 5 Calculated brittleness values and CP of rocks.

\begin{tabular}{lrlll}
\hline Sample No & B1 & B2 & B3 & CP \\
\hline BS-1 & 8.83 & 0.80 & 3712 & 0.78 \\
BS-2 & 10.65 & 0.83 & 3601 & 0.79 \\
BS-3 & 7.30 & 0.76 & 3976.5 & 0.77 \\
BS-4 & 9.81 & 0.81 & 1416.95 & 0.41 \\
BS-5 & 10.32 & 0.82 & 1491.75 & 0.69 \\
BS-6 & 7.87 & 0.77 & 2265.6 & 0.53 \\
BS-7 & 7.75 & 0.77 & 2232 & 0.70 \\
BS-8 & 6.73 & 0.74 & 2831.85 & 0.55 \\
BS-9 & 8.28 & 0.78 & 1825.95 & 0.39 \\
BS-10 & 9.55 & 0.81 & 1910 & 0.66 \\
BS-11 & 7.73 & 0.77 & 2045.85 & 0.66 \\
BS-12 & 9.21 & 0.80 & 1662.5 & 0.69 \\
BS-13 & 8.60 & 0.79 & 3136.05 & 0.74 \\
BS-14 & 10.31 & 0.82 & 3484 & 0.48 \\
BS-15 & 9.05 & 0.80 & 1995 & 0.49 \\
BS-16 & 10.44 & 0.83 & 1692 & 0.48 \\
BS-17 & 10.21 & 0.82 & 1843 & 0.50 \\
BS-18 & 8.65 & 0.79 & 1730 & 0.58 \\
BS-19 & 9.79 & 0.81 & 1767 & 0.62 \\
BS-20 & 9.61 & 0.81 & 1734.7 & 0.62 \\
BS-21 & 7.61 & 0.77 & 1842.5 & 0.42 \\
BS-22 & 8.70 & 0.79 & 1570.35 & 0.41 \\
BS-23 & 8.51 & 0.79 & 1088.8 & \\
\hline
\end{tabular}

On the other hand for basaltic rocks, if volcanic glass and plagioclase is predominant in matrix, rock is being defeated at lower strengths (Erişiş, 2016). For example, BS-2 has the lowest $\mathrm{SiO}_{2}$ value (45.22\%) and the highest UCS value $(277 \mathrm{MPa})$, on the contrary BS-23 has the highest $\mathrm{SiO}_{2}$ value $(60.97 \%)$ and the lowest UCS value (136.3 MPa).

\section{RESULTS AND DISCUSSION}

The following three definitions (Eqns. (1), (2) and (3)) are widely used in previous studies to quantify brittleness indirectly based on strength:

$B 1=\frac{\sigma_{c}}{\sigma_{t}}$

$B 2=\frac{\sigma_{c}-\sigma_{t}}{\sigma_{c}+\sigma_{t}}$

$B 3=\frac{\sigma_{c} * \sigma_{t}}{2}$

where B1, B2, B3 is brittleness index (suggested by Hucka and Das 1974 and Altındag 2002), oc is the uniaxial compressive strength and $\sigma \mathrm{t}$ is Brazilian tensile strength. These formulas have been employed in many studies. Uniaxial compressive strength and Brazilian tensile strength are basic rock mechanical properties and are respectively easy to obtain. And these abovementioned properties can be employed to predict brittleness index when other data are unavailable (Table 5).

Brittle behavior of rocks is related to mineral composition. It is generally assumed that brittle rocks have a high content of brittle minerals which causes an increase in the brittleness. The presence of such minerals (e.g., quartz, dolomite) makes rocks more brittle whereas the presence of clay minerals makes rocks more ductile. As clay content increases, the content of brittle mineralogy along with the geomechanical brittleness indices decreases accordingly. This difference may be the result of the fact that geomechanical properties of rocks are the complex function of both mineralogy and microstructure (Guo et al., 2013). In addition to this, rock properties such as chemical composition can also be used for the quantification of rock brittleness of basaltic rocks.

In this study a chemical parameter (CP) identified considering major oxides of basalt samples. $\mathrm{Al}_{2} \mathrm{O}_{3}, \mathrm{Fe}_{2} \mathrm{O}_{3}$ and $\mathrm{MgO}$ values (show positive linear correlation with B3, Fig. 2) were chosen for the nominator of the formula, while $\mathrm{SiO}_{2}$ values (show negative linear correlation with B3, Fig. 3) were chosen for the denominator (Eqn. (4)). The mineralogical composition of the basalts used in this study is olivine, pyroxene, plagioclase and volcanic glass. Olivine, pyroxene and plagioclase minerals have high $\mathrm{Al}_{2} \mathrm{O}_{3}, \mathrm{Fe}_{2} \mathrm{O}_{3}$ and $\mathrm{MgO}$ content respectively while volcanic glass has high $\mathrm{SiO}_{2}$ content. As regards to new formulization proposed (Eqn. (4)), chemical parameter is decreasing with high $\mathrm{SiO}_{2}$ content which means more brittle behavior while it is increasing with $\mathrm{Al}_{2} \mathrm{O}_{3}, \mathrm{Fe}_{2} \mathrm{O}_{3}$ and $\mathrm{MgO}$ which means more ductile material.

This new chemical parameter (CP) which was proposed for predicting brittleness via major oxide element components of basaltic rocks is presented in Eqn. (4). 
Table 6 Correlation coefficients (R values) for Brittle index, CP with chemical content, mineral content, physical properties and mechanical properties.

\begin{tabular}{lccrc}
\hline Chemical Properties & B1 R value & B2 R value & B3 R value & CP R value \\
\hline $\mathrm{SiO}_{2}$ & 0.30 & 0.34 & -0.80 & -0.98 \\
$\mathrm{Al}_{2} \mathrm{O}_{3}$ & 0.00 & $2 \mathrm{E}-05$ & 0.57 & 0.55 \\
$\mathrm{Fe}_{2} \mathrm{O}_{3}$ & 0.00 & 0.01 & 0.79 & 0.86 \\
$\mathrm{CaO}$ & 0.44 & 0.48 & 0.60 & 0.79 \\
$\mathrm{MgO}$ & 0.24 & 0.27 & 0.70 & 0.87 \\
$\mathrm{~K} 2 \mathrm{O}$ & 0.10 & 0.11 & 0.05 & 0.13 \\
$\mathrm{Na}_{2} \mathrm{O}$ & 0.24 & 0.28 & 0.24 & 0.13 \\
Ignition Loss & 0.15 & 0.14 & $3 \mathrm{E}-05$ & 0.19 \\
\hline Chemical Properties & $\mathrm{B} 1 \mathrm{R}$ value & $\mathrm{B} 2 \mathrm{R}$ value & $\mathrm{B} 3 \mathrm{R}$ value & $\mathrm{CP}$ R value \\
\hline Pyroxene & - & 0.05 & 0.03 & 0.03 \\
Plagioclase & 0.41 & 0.41 & 0.12 & 0.18 \\
Olivine & 0.00 & 0.07 & 0.82 & 0.92 \\
Opaque Mineral & 0.43 & 0.44 & 0.03 & 0.12 \\
Matrix & 0.26 & 0.26 & 0.18 & 0.21 \\
\hline Physical Properties & $\mathrm{B} 1 \mathrm{R}$ value & $\mathrm{B} 2 \mathrm{R}$ value & $\mathrm{B} 3 \mathrm{R}$ value & $\mathrm{CP} \mathrm{R}$ value \\
\hline Unit Weight & 0.17 & 0.20 & 0.79 & 0.83 \\
Porosity & 0.16 & 0.14 & 0.18 & $4 \mathrm{E}-05$ \\
Water Absorbtion & 0.16 & 0.14 & 0.26 & 0.05 \\
P Wave Velocity & 0.14 & 0.18 & 0.88 & 0.92 \\
\hline Mechanical Properties & $\mathrm{B} 1 \mathrm{R}$ value & $\mathrm{B} 2 \mathrm{R}$ value & $\mathrm{B} 3 \mathrm{R}$ value & $\mathrm{CP} \mathrm{R}$ value \\
\hline Point load strength & 0.30 & 0.33 & 0.82 & 0.96 \\
Uniaxial compressive strength & 0.21 & 0.17 & 0.92 & 0.74 \\
Brazilian tensile strength & 0.47 & 0.51 & 0.93 & 0.81 \\
\hline
\end{tabular}

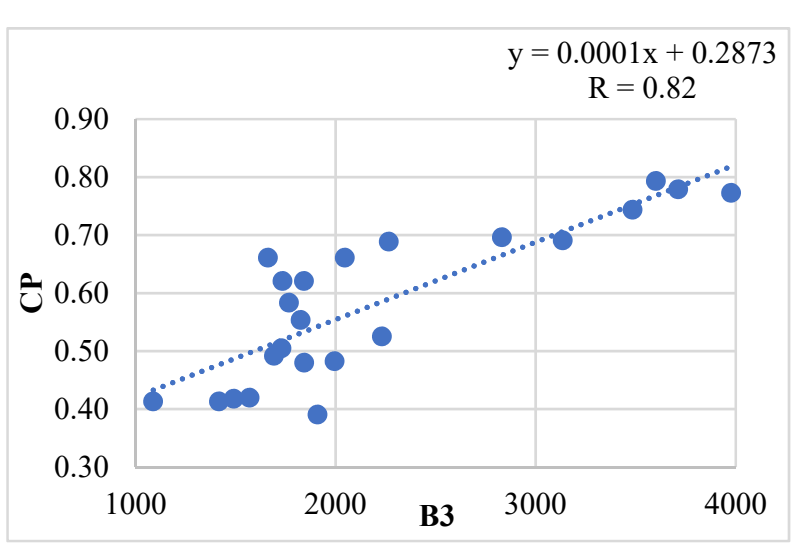

Fig. 4 Correlation between brittleness indices B3-CP.

$\mathrm{CP}=\frac{\mathrm{Al}_{2} \mathrm{O}_{3}+\mathrm{Fe}_{2} \mathrm{O}_{3}+\mathrm{MgO}}{\mathrm{SiO}_{2}}$

Simple regression analysis was used to obtain relationships between brittleness index and geological properties of basaltic rocks. The correlation coefficients $(\mathrm{R})$ were calculated by the least square method and linear functions were considered during these tests (Figs. 2, 3, 4). Since there are no strong correlation coefficients between B1 and B2 and rock properties, the results of regression analysis indicated that the B1 and B2 are not so meaningful for the understanding of the brittle or ductile behavior of the samples investigated.
On the other hand there is a strong relationship, including high correlation coefficient, between the rock properties and B3. For instance, Kaunda and Asbury (2016) determined brittleness using artificial neural networks (ANN) on hard rocks and they found that there were meaningful relationship between P-S wave and elastic properties. In this study $\mathrm{S}$ wave and elastic properties were not studied but the other highest correlation coefficients were calculated with $\mathrm{P}$-wave velocity as 0.88 for B3 (Figs. $2 \mathrm{f}$ and $3 \mathrm{e}$ ).

As a result, linear relationships were obtained between $\mathrm{CP}$ and $\mathrm{Fe}_{2} \mathrm{O}_{3}, \mathrm{CaO}, \mathrm{MgO}$, olivine, unit weight, $\mathrm{P}$ wave velocity, Is (50) point load strength, uniaxial compressive strength and Brazilian tensile strength, respectively. However, only negative linear relation exists between $\mathrm{CP}$ and $\mathrm{SiO}_{2}$ content (Fig. 3).

As regards to Figures $3(\mathrm{a}-\mathrm{j})$, chemical parameter (CP) has significant relations with various parameters analyzed. $\mathrm{R}$ values are tabulated in Table 6 . Accordingly, R-values (for each parameter with respect to $\mathrm{B} 1, \mathrm{~B} 2, \mathrm{~B} 3$ and $\mathrm{CP}$ ) are provided in Table 6; respectively for chemical content, mineral content, physical properties and mechanical properties.

Referring to Table 5, highest $\mathrm{R}$ values (represented in bold) are denoted as very significant and very strong relation. For example, highest $\mathrm{R}$ value is obtained from the plot of $\mathrm{SiO}_{2}$ content and $\mathrm{CP}$ (negative $\mathrm{R}$ value, -0.98). On the other hand, lowest $\mathrm{R}$ values are obtained with "ignition loss" parameter. In this case in terms of brittleness index, $\mathrm{SiO}_{2}$ content should be considered in detail, while it was found out ignition loss is no effective parameter on brittleness. 
Olivine content is also observed to be the one showing significant relationship among others and it resulted as highest $\mathrm{R}$ values such as 0.82 and 0.92 respectively for B3 and CP. As in the same context, Table 6 summarizes the fact that unit weight among physical properties has the highest $\mathrm{R}$ values compared to other physical properties. This abovementioned explanation is only valid for B3 and $\mathrm{CP}$ values, and the corresponding $\mathrm{R}$ values are 0.79 and 0.83 , respectively. In terms of mechanical properties, point load strength, uniaxial compressive strength and Brazilian tensile strength with respect to B3 and CP has very significant $R$ values $(0.93$ for $B 3$ and 0.96 for $\mathrm{CP}$, respectively). In order to represent the correlation between brittleness indices, Figure 4 is provided.

As regards to Figure 4, correlation between B3 and $\mathrm{CP}$ yields a $\mathrm{R}$ value of 0.82 . It is not always possible to evaluate mechanical or physical properties of rocks and that is why it would not be possible to understand brittleness behavior of rocks based on the definitions of B3 (Altindag, 2002) nor based on the brittleness index approaches given in Table 1 . Because of this abovementioned reason, $\mathrm{CP}$ can be proposed to be an alternative way to understand brittleness behavior of rocks. Altindag (2010) has also evaluated brittleness indexes in rock-drilling efficiency, and he pointed out the fact that "there is no standardized and universally accepted brittleness concept". In the same study of Altindag (2010), author has not only pointed out the abovementioned fact but also he stated the fact that there is no available measuring method of rock brittleness. Referring back and forth to the study of Altindag (2010), many researchers (Baron, 1962; Coates and Parsons, 1966; Lawn and Marshall, 1979; Aubertin et al., 1994; Quinn and Quinn, 1997; Ribacchi, 2000; Hajiabdolmajid and Kaiser, 2003) have either introduced brittleness index obtained from stress-strain curves or employed these indexes accordingly. Altindag (2010) has pointed out Andreev (1995) review which has 20 different formulations and definitions of rock brittleness. Although as summarized by Altindag (2010), still no available brittleness index of rocks widely accepted, $\mathrm{CP}$ in this context can be proposed to be a new and novel approach. CP can be employed well in terms of brittleness characterization of rocks and corresponding validation of it is provided either by tabulated $\mathrm{R}$ values or the corresponding graphical demonstrations (Figure 3 and Figure 4). And in this context, for basalt type rocks in specific, this new approach for brittleness index prediction (CP) is claimed to be better successful than any other approaches proposed previously.

Rock brittleness as it is described by Zhang et al. (2021) is affected by internal and external factors and mineral composition of rocks is one of the primary internal factor affecting the rock brittleness. Mineral composition of rocks changes with their chemical composition so does the rock brittleness. Although brittleness is mostly evaluated based on stress-strain curve, special tests, mineral composition \& porosity,
Mohr Envelope (Zhang et al., 2021), differentiation in brittleness index values could have been related to differentiation of chemical composition for the specific rock samples, i.e. basalt samples in this case. Meng et al. (2021) have reviewed 11 groups of brittleness indices in their study and chemical composition or chemical parameter was not even partly considered. Not only brittleness indices based on mineral contents ( $\mathrm{B}_{48}$ to $\mathrm{B}_{54}$ from the study of Meng et al., 2021) can be regarded as ignoring the important factors (stress state, diagenesis, pre-consolidation pressure, porosity, grain size, type and strength of cementing material) contributing the rock brittleness (Meng et al., 2021), but also it can be claimed that they (brittleness indices based on mineral contents) overlay the importance of chemical composition in this regard. Mineral and chemical composition of basalts are generally questioned together as in the study of Aal (1998) and Babievskaya et al. (2009) have proposed a method to calculate mineral composition of basaltic rocks based on their chemical composition. So, referring abovementioned researchers (Aal, 1998 and Babievskaya et al., 2009) once again, chemical composition of basaltic rocks should be as significant as mineral composition in terms of brittleness behavior evaluation.

\section{CONCLUSION}

In this study, suggested CP formula provided very high strength correlation coefficients (Table 5) accordance with the $\mathrm{SiO}_{2}$ (R: -0.98), olivine (R: 0.92), $P$ wave velocity (R: 0.92), Is ${ }_{(50)}(\mathrm{R}: 0.96)$, in addition to it has high strength correlation coefficients accordance with $\mathrm{Fe}_{2} \mathrm{O}_{3}$ (R: 0.86), $\mathrm{CaO}$ (R: 0.79), $\mathrm{MgO}$ (R: 0.87), unit weight (R: 0.83), UCS (R: 0.74), BTS (R: 0.81) (Table 5). By taking these strong correlation coefficients into account, it is thought that the newly suggested chemical parameter (CP) formula will be useful to determination and prediction of rock brittleness if chemical content of rock is known. In this way different properties of basaltic rocks could be predicted with the help of this formula (CP). The traditional methods for strength measurement require core samples, which are expensive and are time consuming; and maintaining the in-situ condition and sample preparation are effortful circumstances. So the use of major oxide-based on $\mathrm{CP}$ for predicting of basaltic rocks brittleness behavior, instead of strength-based B3 $(\mathrm{R}=0.82)$ brittleness index is highly reliable.

It is concluded that, B1 and B2 are not reliable parameters for predicting the different properties however, B3 and CP can be used as good criteria for predicting the different properties of basaltic rocks (especially chemical and mechanical properties).

\section{REFERENCES}

Aal, A.Y.A.: 1998. Mineral and chemical composition of basalts in the neighbourhood of Giza, Egypt. J. Afr. Earth Sci., 26, 1, 101-117. DOI: 10.1016/S0899-5362(97)00139-5 
Ai, C., Zhang, J., Li, Y.W., Zeng, J., Yang, X.L. and Wang, J.G.: 2016, Estimation criteria for rock brittleness based on energy analysis during the rupturing process. Rock Mech. Rock Eng., 49, 4681-4698. DOI: $10.1007 / \mathrm{s} 00603-016-1078-\mathrm{x}$

Aligholi, S., Lashkaripour, G.R. and Ghafoori, M.: 2017, Strength/brittleness classification of igneous intact rocks based on basic physical and dynamic properties. Rock Mech. Rock Eng., 50, 45-65. DOI: $10.1007 / \mathrm{s} 00603-016-1106-\mathrm{x}$

Altindag, R.: 2000, The role of rock brittleness on the analysis of percussive drilling performance (in Turkish). In: Proceedings the Fifth Turkish National Rock Mechanics Symposium, Isparta, Turkey, 105112.

Altindag, R.: 2002, The evaluation of rock brittleness concept on rotary blast hole drills. J. South African Inst. Min. Metall., 102, 1, 61-66.

Altindag, R.: 2003, The correlation of specific energy with rock brittleness concept on rock cutting. J. South African Inst. Min. Metall., 103, 163-171.

Altindag, R.: 2010, Assessment of some brittleness indexes in rock-drilling efficiency. Rock Mech. Rock Eng., 43, 3, 361-370. DOI: 10.1007/s00603-009-0057-x

Altindag, R. and Guney, A.: 2010, Predicting the relationships between brittleness and mechanical properties (UCS, TS and SH) of rocks. Sci. Res. Essays, 5, 16, 2107-2118.

DOI: $10.5897 /$ SRE. 9000753

Andreev, G.E.: 1995, Brittle failure of rock materials: test results and constitutive models. A. A. Balkema, Rotterdam.

American Society for Testing and Materials (ASTM): 2001, Standard test method for splitting tensile strength of intact rock core specimens. D3967. 95(a).

American Society for Testing and Materials (ASTM): 2010, Standard test method for compressive strength and elastic module of intact rock core specimens under varying states of stress and temperatures D7012.

Aubertin, M., Gill, D.E. and Simon, R.: 1994, On the use of the brittleness index modified (BIM) to estimate the post-peak behaviour of rocks. 1st North American Rock Mechanics Symposium, June 1-3, 1994, Austin, Texas, 945-952.

Babievskaya, I.Z., Drobot, N.F., Fomichev, S.V. and Krenev, V.A: 2009, Calculation of the mineral composition of basaltic rocks. Inorg. Mater., 45, 8, 916-918.

Baron, L.I.: 1962, Determination of properties of rocks. Gozgotekhizdat, Moscow, (in Russian).

Bishop, A.W.: 1967, Progressive failure with special reference to the mechanism causing it. Proc. Geotechnical conference, Oslo, 142-150.

Chen, G.Q., Jiang, W.Z., Sun, X., Zhao, C. and Qin, C.A.: 2019a, Quantitative evaluation of rock brittleness based on crack initiation stress and complete stressstrain curves. Bull. Eng. Geol. Environ., 78, 59195936. DOI: $10.1007 / \mathrm{s} 10064-019-01486-2$

Chen, J., Zhang, G., Chen, H. and Yin, X.: 2014, The construction of shale rock physics efective model and prediction of rock Brittleness. SEG Annual Meeting, Society of Exploration Geophysicists, Denver, 28612865. DOI: 10.1190/segam2014-0716.1

Coates, D.F. and Parsons, R.C.: 1966, Experimental criteria for classification of rock substances. Int. J. Rock Mech. Min. Sci. Geomech. Abstr., 3, 181-189. DOI:10.1016/0148-9062(66)90022-2
Copur, H., Bilgin, N., Tuncdemir, H. and Balci, C.: 2003, A set of indices based on indentation tests for assessment of rock cutting performance and rock properties. J. South African Inst. Min. Metall., 103, 9, 589-599.

Erişiş, S.: 2016, The effect of composition and textural properties on the mechanical behaviours of basaltic rocks. M.Sc Thesis, İstanbul University.

Gong, Q.M. and Zhao, J.: 2007, Influence of rock brittleness on TBM penetration rate in Singapore granite tunnel. Tun. Undergr. Sp. Tech., 22, 3, 317-324. DOI: $10.1016 /$ j.tust.2006.07.004

Guo, Z., Chapman, M. and Li, X.: 2012, Exploring the efect of fractures and microstructure on brittleness index in the Barnett Shale. SEG Annual Meeting, Society of Exploration Geophysicists, Las Vegas, 4609-4613. DOI: 10.1190/segam2012-0771.1

Guo, Z., Li, X.Y., Liu, C., Feng, X. and Shen, Y.: 2013, A shale rock physics model for analysis of brittleness index, mineralogy, and porosity in the Barnett Shale. J. Geophys. Eng., 10, 2, 1-10. DOI: $10.1088 / 1742-2132 / 10 / 2 / 025006$

Hajiabdolmajid, V. and Kaiser, P.: 2003, Brittleness of rock and stability assessment in hard rock tunnelling. Tun. Undergr. Sp. Tech., 18, 1, 35-48. DOI: $10.1016 / \mathrm{S} 0886-7798(02) 00100-1$

Huang, X.R., Huang, J.P., Li, Z.C., Yang, Q.Y., Sun, Q.X. and Cui, W.: 2015, Brittleness index and seismic rock physics model for anisotropic tight-oil sandstone reservoir. Appl. Geophys., 12, 1, 11-22. DOI: $10.1007 / \mathrm{s} 11770-014-0478-0$

Hucka, V. and Das, B.: 1974, Brittleness determination of rocks by different methods. Int. J. Rock Mech. Min. Sci., 11, 10, 389-392. DOI: 10.1016/01489062(74)91109-7

Ingram, G.M. and Urai, J.L.: 1999, Top-seal leakage through faults and fractures: the role of mudrock properties. Geol. Soc. Lond., 158, 1, 125-135.

DOI: 10.1144/GSL.SP.1999.158.01.10

International Society for Rock Mechanics (ISRM): 2007, The complete ISRM suggested methods for rock characterization, testing and monitoring: 1974-2006. In: Ulusay, R. and Hudson, J.A. (eds), Suggested methods prepared by the commission on testing methods, ISRM, Compilation arranged by the ISRM Turkish National Group, Kozan ofset, Ankara.

Jarvie, D.M., Hill, R.J., Ruble, T.E. and Pollastro, R.M.: 2007, Unconventional shale-gas systems: the Mississippian Barnett Shale of northcentral Texas as one model for thermogenic shale-gas assessment. AAPG Bull., 9, 4, 475-499.

DOI: $10.1306 / 12190606068$

Jin, X., Shah, S.N., Truax, J.A. and Roegiers, J.C.: 2014, A practical petrophysical Ap- proach for brittleness prediction from porosity and sonic logging in shale reservoirs. In: SPE (Society of Petroleum Engineers) Annual Technical Conference and Exhibition, October 27-29, Amsterdam, The Netherlands.

Kahraman, S.: 2002, Correlation of TBM and drilling machine performance with rock brittleness. Eng. Geol., 65, 4, 269-283. DOI: $10.1016 / \mathrm{S} 0013-7952(01) 00137-5$

Kahraman, S. and Atindag, R.: 2004, A brittleness index to estimate fracture toughness. Int. J. Rock Mech. Min. Sci., 41, 2, 343-347.

DOI: $10.1016 /$ j.ijrmms.2003.07.010 
Kaunda, R.B. and Asbury, B.: 2016, Prediction of rock brittleness using nondestructive methods for hard rock tunnelling. J. Rock Mech. Geotech. Eng., 8, 4, 533540. DOI: $10.1016 /$ j.jrmge.2016.03.002

Korkanç, M.: 2003, Evaluation of Niğde basalts as alternative aggregate. Ph.D dissertation, İstanbul University.

Korkanç, M. and Tugrul, A.: 2004, Evaluation of selected basalts from Niğde, Turkey, as source of concrete aggregate. Eng. Geol., 75, 3-4, 291-307. DOI: 10.1016/j.enggeo.2004.06.015

Korkanç, M. and Tugrul, A.: 2005, Evaluation of selected basalts from the point of alkali-silica reactivity. Cement Concrete Res., 35, 3, 505-512.

DOI: 10.1016/j.cemconres.2004.06.013

Lawn, B.R. and Marshall, D.B.: 1979, Hardness, toughness, and brittleness: an indentation analysis. J. Am. Ceram. Soc., 62, 347-350. DOI: $10.1111 / j .1151-2916.1979 . t b 19075 . x$

LeBas, M.J. and Streckeisen, A.L.: 1991, The IUGS systematics of igneous rocks. J. Geol. Soc., 148, 825833. DOI: $10.1144 /$ gsjgs. 148.5.0825

Luan, X.Y., Di, B.R., Wei, J.X., Li, X.Y., Qian, K.R., Xie, J.Y. and Ding, P.B.: 2014, Laboratory measurements of brittleness anisotropy in synthetic shale with diferent cementation. SEG Annual Meeting, Society of Exploration Geophysicists, Denver, 3005-3009. DOI: $10.1190 /$ segam2014-0432.1

Meng, F., Zhou, H., Zhang, C., Xu, R. and Lu, J.: 2015, Evaluation methodology of brittleness of rock based on post-peak stress-strain curves. Rock Mech. Rock Eng., 48, 1787-1805. DOI: 10.1007/s00603-0140694-6

Meng, F., Wong, L.N.Y. and Zhou, H.: 2021, Rock brittleness indices and their applications to different fields of rock engineering: A review. J. Rock Mec. Geotech. Eng., 13, 1, 221-247. DOI: 10.1016/j.jrmge.2020.06.008

Mikaeil, R., Ataei, M. and Yousefi, R.: 2013, Correlation of production rate of ornamental stone with rock brittleness indexes. Arab. J. Geosci., 6, 115-121. DOI: 10.1007/s12517-011-0311-x

Militky, J. and Kovacic, V.: 1996, Ultimate mechanical properties of basalt filaments. Textile Res. J., 66, 225229. DOI: $10.1177 / 004051759606600407$

Nygård, R., Gutierrez, M., Bratli, R.K. and Høeg, K.: 2006, Brittle-ductile transition, shear failure and leakage in shales and mudrocks. Mar. Pet. Geol., 23, 2, 201-212. DOI: 10.1016/j.marpetgeo.2005.10.001

Park, J.M., Shin, W.G. and Yoon, D.J.: 1999, A study of interfacial aspects of epoxy-based composites reinforced with dual basalt and $\mathrm{SiC}$ fibres by means of the fragmentation and acoustic emission techniques. Compos. Sci. Technol., 59, 355-370. DOI: $10.1016 / \mathrm{S} 0266-3538(98) 00085-2$

Protodyakonov, M.M.: 1962, Mechanical properties and drillability of rocks. Proc. 5th Symposium on Rock Mechanics. University of Minnesota, Minneapolis, Minnesota, 103-118.

Quinn, J.B. and Quinn, G.D.: 1997, Indentation brittleness of ceramics: a fresh approach. J. Mater. Sci., 32, 43314346.

Reichmuth, R.D.: 1968, Point load testing of brittle materials to determine tensile strength and relative brittleness. Proc. 9th Symposium on Rock Mechanics (USRMS), April 17-19, Golden Colorado, 134-159.
Rickman, R., Mullen, M., Petre, E., Grieser, B. and Kundert, D.: 2008, A practical use of shale petrophysics for stimulation design optimization: all shale plays are not clones of the Barnett Shale. SPE Annual Technical Conference and Exhibition. Society of Petroleum Engineers, SPE-115258. DOI: 10.2118/115258-MS

Ribacchi, R.: 2000, Mechanical tests on pervasively jointed rock material: Insight into rock mass behavior. Rock Mech. Rock Eng., 33, 4, 243-266.

DOI: $10.1007 / \mathrm{s} 006030070002$

Tarasov, B. and Potvin, Y.: 2013, Universal criteria for rock brittleness estimation under triaxial compression. Int. J. Rock Mech. Min. Sci., 59, 57-69. DOI: 10.1016/j.ijrmms.2012.12.011

Wang, Y., Li, X., Wu, Y.F., Fen, Y.X., Li, D.D. and He, J.M.: 2014, Research on relationship between crack initiation stress level and brittleness indices for brittle rocks. Chin. J. Rock Mech. Eng., 33, 2, 265-275, (in Chinese)

Xia, Y.J., Li, L.C., Tang, C.A., Ma, S., Li, M. and Bao, C.Y.: 2016, Rock brittleness evaluation based on stress dropping rate after peak stress and energy ratio. Chin. J. Rock Mech. Eng., 35, 6, 1141-1154, (in Chinese).

Xia, Y.J., Li, L.C., Tang, C.A., Li, X.Y., Ma, S. and Li, M.: 2017, A new method to evaluate rock mass brittleness based on stress-strain curves of class I. Rock Mech. Rock Eng., 50, 1123-1139. DOI: $10.1007 / \mathrm{s} 00603-017-1174-6$

Yagiz, S.: 2006, An investigation on the relationship between rock strength and brittleness. 59th Geological Congress of Turkey, MTA General Directory, March 20-24, Ankara, Turkey, Abstract, 352.

Yagiz, S.: 2009, Assessment of brittleness using rock strength and density with punch penetration test. Tunn. Undergr. Sp. Tech., 24, 1, 66-74. DOI: $10.1016 /$ j.tust.2008.04.002

Yagiz, S. and Gokceoglu C.: 2010, Application of fuzzy inference system and nonlinear regression models for predicting rock brittleness. Expert Syst. Appl., 37, 3, 2265-2272. DOI: 10.1016/j.eswa.2009.07.046

Yagiz, S. and Karahan H.: 2015, Application of various optimization techniques and comparison of their performances for predicting TBM penetration rate in rock mass. Int. J. Rock Mech. Min. Sci., 80, 308-315. DOI: 10.1016/j.ijrmms.2015.09.019

Zhang, D., Ranjith, P.G. and Perera, M.S.A.: 2016, The brittleness indices used in rock mechanics and their application in shale hydraulic fracturing: A review. J. Pet. Sci. Eng., 143, 158-170. DOI: $10.1016 /$ j.petrol.2016.02.011

Zhang, Y., Feng, X.T., Yang, C., Han, Q., Wang, Z. and Kong, Z.: 2021, Evaluation method of rock brittleness under true triaxial stress states based on pre-peak deformation characteristic and post-peak energy evolution. Rock Mech. Rock Eng., 54, 1277-1291. DOI: $10.1007 / \mathrm{s} 00603-020-02330-\mathrm{w}$

Zhou, H., Chen, J., Lu, J., Jiang, Y. and Meng, F.: 2018, A new rock brittleness evaluation index based on the internal friction angle and class I stress-strain curve. Rock Mech. Rock Eng., 51, 2309-2316. DOI: $10.1007 / \mathrm{s} 00603-018-1487-0$ 